\title{
Alignment of the Near Detector Scintillator Modules Using Cosmic Ray Muons
}

\author{
Rustem Ospanov and Karol Lang \\ Department of Physics \\ 1 University Station C1600 \\ The University of Texas at Austin \\ Austin, TX 78712
}

August 12, 2005

\begin{abstract}
We describe the procedures and the results of the first alignment of the Near Detector. Using 15.5 million cosmic ray muon tracks, collected from October, 2004 through early January, 2005, we derive the effective transverse positions of the calorimeter scintillator modules. The residuals from straight line fits indicate that the current alignment has achieved better than $1 \mathrm{~mm}$ precision. We estimate the size of the remaining misalignment and using tracks recorded with a magnetic field test the effect of the magnetic field on the alignment.
\end{abstract}




\section{Contents}

1 Introduction $\quad 3$

2 Alignment procedure $\quad 3$

3 Cosmic ray muon data $\quad 5$

4 Alignment results $\quad 5$

5 Alignment stability $\quad 11$

6 Comparison with the independent data set $\quad 11$

$\begin{array}{lll}7 & \text { Plane rotations } & 12\end{array}$

8 Alignment consistency checks $\quad 14$

9 Conclusions $\quad 18$

$\begin{array}{lll}\text { Appendix A } & \text { Dependence of alignment on defective channels } & 19\end{array}$

$\begin{array}{lll}\text { Appendix B } & \text { Dependence of track reconstruction on alignment } & 22\end{array}$ 


\section{Introduction}

The installation of the MINOS Near Detector started in March, 2004 and was completed on August 11th, 2004. The magnet coil of the Near Detector was powered for the first time on December 9th, 2004, magnetizing the detector steel.

The Near Detector ${ }^{1}$ is constructed from alternating planes of steel and scintillator strips mounted on the steel. Strips are made from polystyrene and are $4.1 \mathrm{~cm}$ wide and $1.0 \mathrm{~cm}$ thick, with lengths varying between $1.0 \mathrm{~m}$ and $3.6 \mathrm{~m}$. All strips in a plane are parallel to each other. Groups of 20 or 24 strips are held together by aluminum casing and form scintillator modules which were individually mounted on the steel plates. Scintillator planes have either 64 strips covering the target part of the calorimeter (these are called "partial planes"), or 96 strips covering most of the steel area (these are called "full planes"). Every fifth calorimeter plane and all spectrometer planes have the full scintillator coverage. Strips in two neighboring planes have orthogonal orientations, called U and V views. Steel planes are single piece elements $2.54 \mathrm{~cm}$ thick.

The detector alignment is necessary for the correct reconstruction of physics events. It is initially assumed that the detector is built exactly according to the design specifications. Using straight tracks from cosmic ray muons in an unmagnetized detector, our alignment procedure determined the actual module placements. The module transverse positions were adjusted iteratively in three iterations.

\section{Alignment procedure}

There are three complimentary sets of data describing the positions of scintillator strips and modules. All three should be used for the complete understanding of the detector alignment.

First, there are maps of the strip positions within the scintillator modules, as measured with the radioactive source mapping station at ANL during the module construction. The ideal module geometry consists of closely packed parallel strips with a strip width of $4.10 \mathrm{~cm}$, and an average gap of $0.1 \mathrm{~cm}$ between the strips. The actual placement of strips within a module may be off by at most a millimeter (as was estimated for the Far Detector modules). At the time when this work is done, the mapping station data for the Near Detector scintillator modules were not yet processed. We assume that strip misplacements within a module, relative to the module's center, are much smaller than the observed misalignments of the modules as a unit.

The second set of data is based on measurements of the scintillator module positions with the laser tracker system ${ }^{2}$ during the detector installation. Similar measurements for the Far Detector modules are accurate to within about a millimeter.

The third approach is to calculate relative offsets of the strip positions using cosmic ray muons in an unmagnetized detector. The alignment of the Near Detector using this approach is the subject of this note. The Near Detector alignment procedure evolved from the similar work done for the Far Detector $^{3}$. During the commissioning stage, the Near Detector collected large amount of cosmic ray muon data. The 463 hours of data used for the alignment span 3 months, from early October of 2004 through early January of 2005. Cosmic ray muons reconstructed before the current in the coil was turned on for the first time on December 9th, 2004, have straight tracks which, due to their high average momentum, suffer from only minor multiple Coulomb scattering. Such scattering does not introduce systematic offsets and only broadens residual distributions.

There are two global detector coordinate systems that are used for the alignment: XYZ system and UVZ system (see Figure 1). The global UVZ and XYZ coordinate systems share the Z-axis which is perpendicular to the detector planes.

In the MINOS reconstruction software, the detector geometry is constructed from the four types of elements: steel plane, scintillator plane, scintillator module and scintillator strip (in the order of

\footnotetext{
${ }^{1}$ The MINOS Detectors, Technical Design Report, Fermilab, NuMI-L-337, Fermilab, October, 1998

${ }^{2}$ D. Boehnlein, NuMI note 878

${ }^{3}$ B. Becker NuMI notes 856, B. Viren NuMI note 876, B. Becker and D. Boehnlein NuMI note 1047
} 


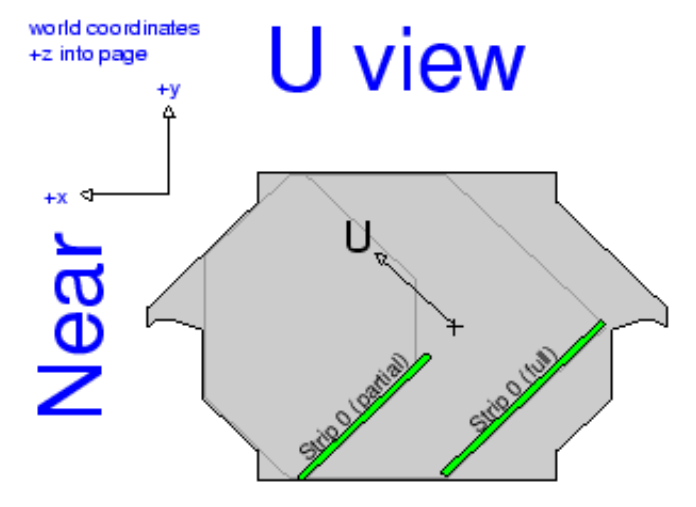

\section{V view}

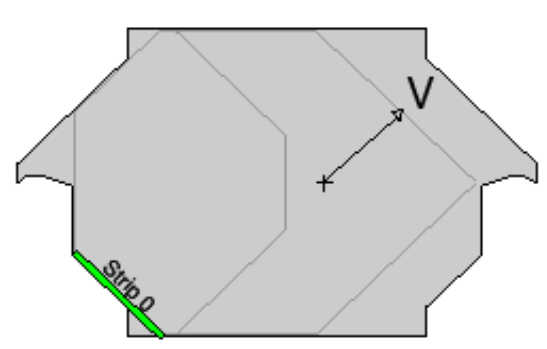

Figure 1: Global Near Detector UVZ and XYZ coordinate systems.

decreasing hierarchy). There is a local XYZ coordinate system for an element of each type ${ }^{4}$. The position of the center and the orientation of the element's local coordinate system are given relative to the local coordinate system of the higher hierarchy element ${ }^{5}$. During the alignment, we do not change positions of the steel and scintillator planes. The centers of modules' coordinate systems are moved along the transverse coordinate $\operatorname{axis}^{6}$. The local positions of the scintillator strips, relative to the center of the module containing strips, are not changed. But the global positions of the strips are determined using the coordinate system of the modules containing them. So the corrections to the global module's position also adjust the global positions of the strips contained in the module.

For simplicity, we use the following approximations about the detector geometry:

1. Strips are orthogonal to the Z-axis

2. Strips are placed into their design positions along Z-axis

3. Strips are exactly parallel to either $\mathrm{U}$-axis or $\mathrm{V}$-axis

We do not have estimates for the sizes of approximations 1 and 2. In Section 7, we give quantitative estimates for the size of approximation 3.

A two dimensional (2d) track is a projection of a three dimensional muon track to either the $\mathrm{U}$ or $\mathrm{V}$ coordinate axis and the Z-axis. It is a collection of all active strips (channels with non zero recorded charge) that are attributed to a track and which are all parallel to the same coordinate axis. The explicit assumption is that in unmagnetized steel $2 \mathrm{~d}$ tracks in $\mathrm{U}$ and $\mathrm{V}$ views can be treated independently. In order to determine residuals, each $2 \mathrm{~d}$ track is fitted with a straight line using the least squares method. A residual is the distance between the transverse position at the center of the strip and the best fit line at the plane's $\mathrm{Z}$ position. To calculate a residual for a given strip, all strips in the $2 \mathrm{~d}$ track are used for the fit except for the current strip.

The alignment is done using the DetectorAlignment software package. This package is loosely based on Brett Viren's Alignment code ${ }^{7}$ and is included in the MINOS offline software repository, where it is a subdirectory of the Alignment package.

The geometry corrections for one alignment iteration are done as follows:

\footnotetext{
${ }^{4}$ For the steel and scintillator planes, the unperturbed local coordinate system is aligned with the global XYZ coordinate system, except for the shifts of the origin along the Z-axis. For strips and modules, the center of the local coordinate system is located at the geometric center of a strip or a module.

${ }^{5}$ For example, position of an origin of the strip's XYZ coordinate system is given relative to the module's XYZ coordinate system

${ }^{6} \mathrm{U}$ and $\mathrm{V}$ view modules are moved along the $\mathrm{U}$-axis and $\mathrm{V}$-axis respectively (Figure 1).

${ }^{7}$ Brett Viren, NuMI note 876
} 
1. Find all strips that form a muon track using initial detector geometry and the standard MINOS reconstruction software, and calculate residuals for all strips on a track ${ }^{8}$

2. Using entire data set of cosmic ray muons, calculate the mean residual offset for each module ${ }^{9}$

3. Adjust transverse positions of the calorimeter modules by the mean module residuals

The muon tracks must be always reconstructed with the new geometry because the new strip positions are used by the track finding algorithm.

We explicitly have turned off all calibration schemes. This might introduce a systematic bias to the alignment results due to the differences in PMT gain, fiber length, and scintillator response. At the time when the alignment has been done, no validated detector calibration existed for the Near Detector.

\section{Cosmic ray muon data}

We use 15.5 million muon tracks recorded over 463 hours $^{10} .414$ hours of the data are collected before the detector steel is magnetized for a first time. Additional 49 hours of the data are collected after the magnet coil is energized, but with the magnet coil off during the data taking. Because the steel is not degaussed, there is some unknown residual magnetic field in the detector. Bending of muon tracks in the magnetic field introduces systematic bias to the calculations of residuals. By comparing the mean residuals between different runs, we conclude that the residual magnetic field in $10 \%$ of our data does not introduce significant alignment errors (see Section 5).

The cosmic ray muon data in the Near Detector are collected using an $\mathrm{M}$ out of $\mathrm{N}$ trigger. $\mathrm{M}$ is a minimum number of plane hits in $\mathrm{N}$ detector planes. A plane hit is defined as PMT dynode signal which is over the $1 / 3$ photoelectron threshold and at least one channel in the plane reads at least one bucket above the sparsification threshold ${ }^{11}$. If the trigger condition is passed, the data acquisition system reads out all hit planes. In those planes, strips with signals above sparsification threshold are readout for at most 8 buckets. In Figure 2 are shown the directional cosines of the three dimensional muon tracks as recorded by the detector. Also shown is the distribution of the length of the muon tracks inside the detector. The track angular distributions are determined both by the flux of the cosmic ray particles and by the geometrical detector acceptance. For example, in Figure 2, the dip around $\mathrm{Z}$ equal 0 , in the plot of cosine of track angle with $\mathrm{Z}$-axis, is due to the trigger acceptance: tracks with cosine near 0 are traveling vertically downward and thus do not cross the required minimum number of planes. See Appendix B for the distributions of $2 \mathrm{~d}$ track charge, number of strips and track fit result.

\section{Alignment results}

Our alignment procedure only adjusts the relative transverse positions of the calorimeter scintillator modules. Further work is required to align strips within the modules and to correct for the rotations of

\footnotetext{
${ }^{8}$ The DetectorAlignmentModule module gets all CandStrips that belong to a reconstructed muon track from the CandTrackSR module. These strips are separated into $U$ view and V view strips to form $2 \mathrm{~d}$ tracks. The $2 \mathrm{~d}$ tracks are fit with a straight line. The fit results and track information are saved to a root file in NtpAlignmentRecord tree. There is a new root file for each subrun of cosmics data. Processing of all data in step 1 requires running 463 independent jobs, and we used Fermilab LSF batch system for the processing.

${ }^{9}$ The MakeAlignmentModule module reads residuals for all 15.5 million muon tracks from 463 separate files in a single job. Tracks that pass cuts are then used to calculate mean residuals for strips and modules. For each strip, a histogram is filled with residuals calculated using tracks that pass through it. Similarly, for each module, a histogram is filled with residuals of all strips in the module.

${ }^{10}$ We only select records (snarls) that have one reconstructed muon track.

${ }^{11}$ It gets more complicated for spectrometer planes. Please refer to the Near Detector data acquisition manual for the details.
} 

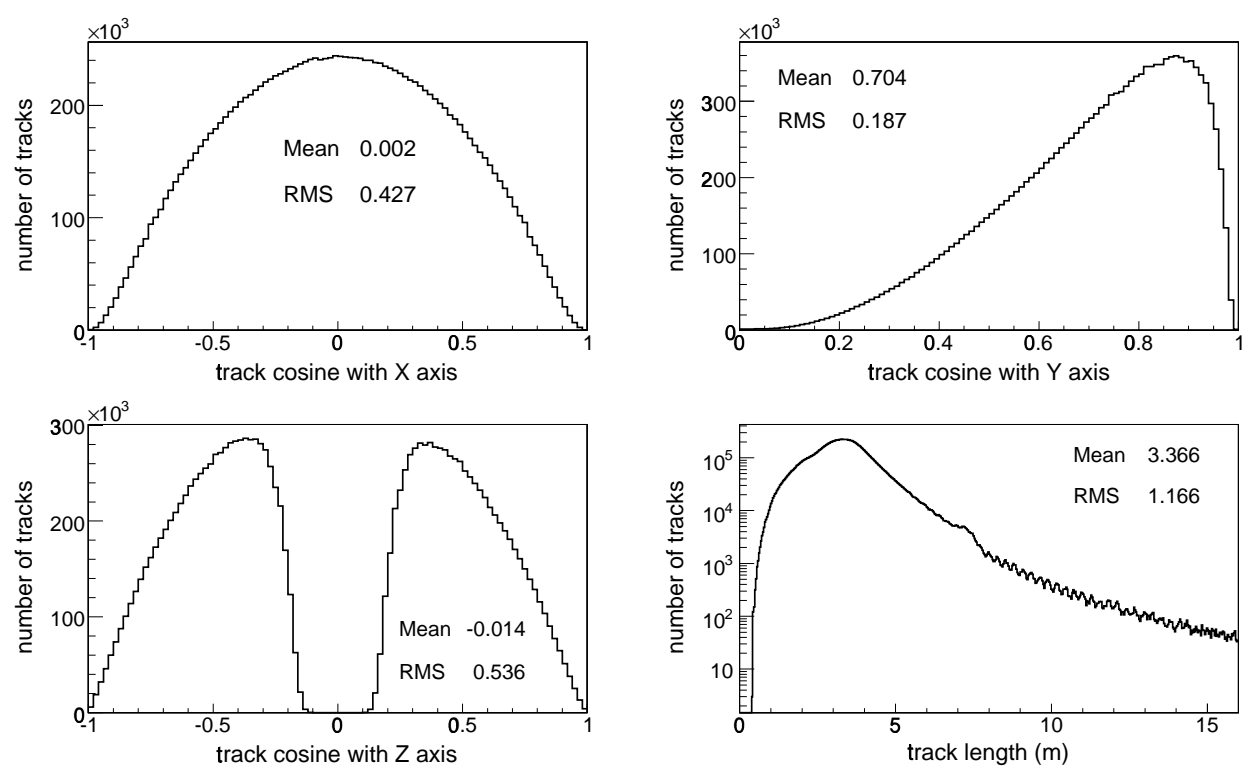

Figure 2: Direction and length distributions for 15.5 million 3d muon tracks as calculated by the DetectorAlignment package.
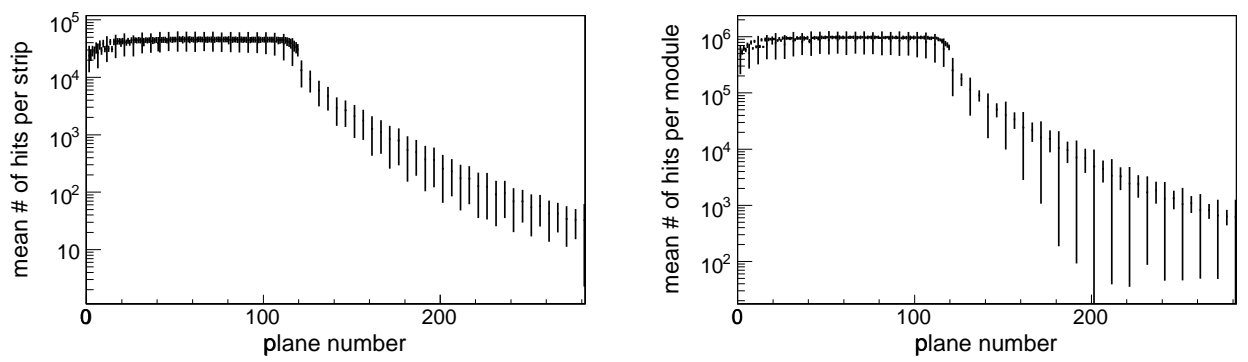

Figure 3: The mean number of muon tracks hits per strip (left) and module (right) versus plane number. The mean is over strips or modules in a plane and the error bars are the sigmas over the strips or modules in a plane.
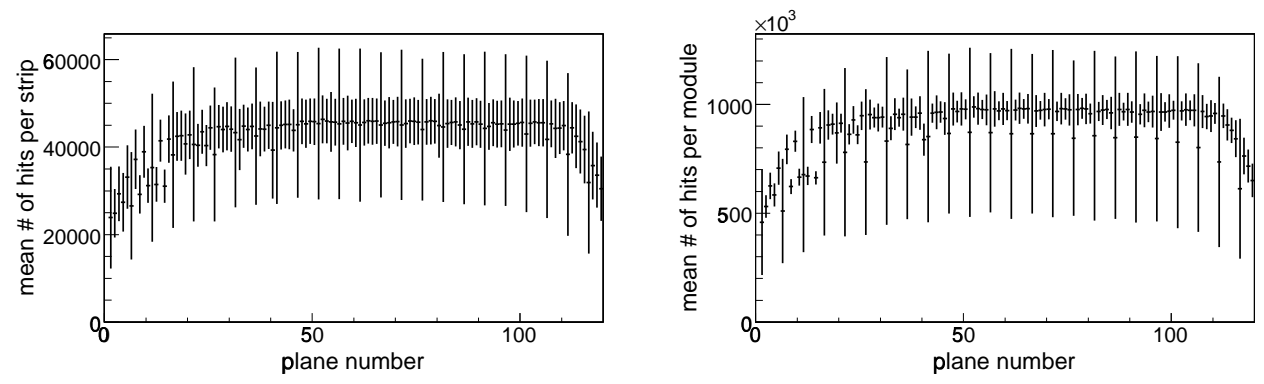

Figure 4: The mean number of muon tracks hits per strip (left) and module (right) versus plane number for calorimeter planes only. The mean is over strips or modules in a plane and the error bars are sigma over strips or modules in a plane. 
the scintillator modules around the Z-axis. We work under an assumption that these effects are smaller than the corrections described in this section. Because of the inconsistencies in track reconstruction ${ }^{12}$ for tracks in the spectrometer part of the detector, none of the spectrometer modules are aligned.
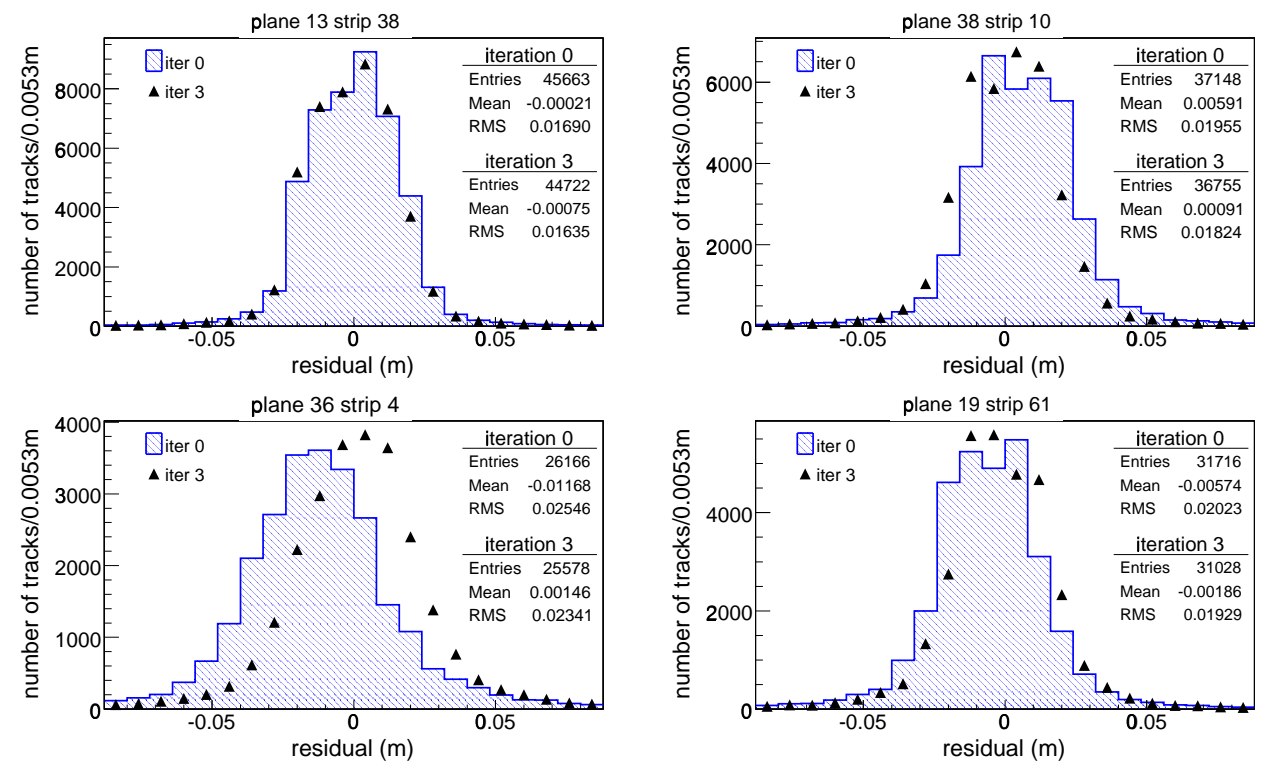

Figure 5: Four random examples of the residual histograms for calorimeter strips before (iteration 0 ) and after (iteration 3) alignment. One histogram entry is one muon track residual. The residual entries in these histogram are also entered for the residual histograms for the modules containing these strips.

We apply five cuts to select the tracks that are used to calculate the mean residual offsets for the calorimeter scintillator modules. These cuts remove 6.3 million muon tracks and the remaining 9.2 million tracks are used for the alignment. The muon tracks have to satisfy these five cuts:

1. 4000 ADC $<$ muon track charge $<60000$ ADC (Figure 36)

2. RMS of track strips' positions ${ }^{13}<0.03 \mathrm{~m}$ (Figure 37 )

3. Ratio of muon track charge over total snarl charge $>0.5$ (Figure 30 )

4. Muon track cosine with the Z-axis $>0.25$ (Figure 2)

5. Maximum strip charge $<5000$ ADC (Figure 22)

These cuts are based on the empirical arguments and are designed to remove tracks that have errors in reconstruction, are too short or are subject to significant multiple Coulomb scattering. In left plot of Figure 9, we show the differences between the mean strip residuals that are calculated with and without these cuts. The plot on the right shows similar differences for the modules. Because these differences are small we conclude that the cuts are not required for the alignment. The alignment corrections are calculated using the tracks that pass all cuts, but the plots in this note are made using entire set of the muon tracks, unless specifically mentioned otherwise. We are allowed to do this since the differences in the mean strip and module residuals calculated with and without cuts are smaller

\footnotetext{
${ }^{12}$ We are using MINOS offline software version R1.13.

${ }^{13}$ This is the square root of the least squares sum, calculated with the best fit parameters (or minimum of the least squares sum). This quantity estimates the variation of strip positions around the best fit line.
} 

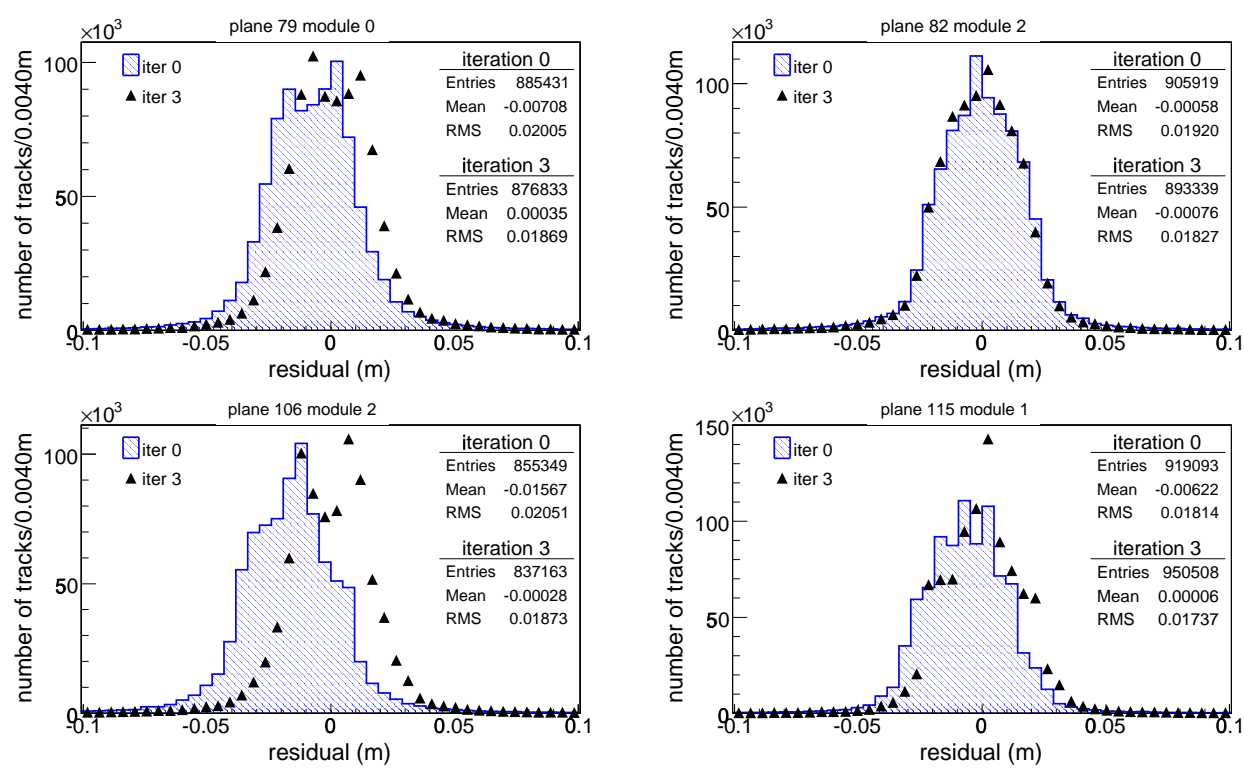

Figure 6: Four random examples of the residual histograms for calorimeter modules before (iteration 0) and after (iteration 3) alignment. One histogram entry is one muon track residual for a strip contained in the module. The mean of the these histograms is used for adjusting the module transverse positions.

than $0.5 \mathrm{~mm}$. In the hindsight, we were not required to use such strict cuts, since they have little effect on the alignment results.

In Figure 7, the left plots show mean module residuals for the calorimeter modules. The strip's coordinates are determined relative to the global position of the module's center, so adjusting the module's coordinates also moves the scintillator strips contained in the module. Figure 8 shows changes in the strip mean residuals. Improved module alignment also improves the alignment of the scintillator strips, because the tracking software uses the new adjusted strip coordinates for the track finding. The gradual improvement of the alignment with each iteration suggests that the alignment procedure is converging to the more precise detector geometry.

The RMS of the mean module residuals is equal to $6.6 \mathrm{~mm}$ before alignment and to $0.275 \mathrm{~mm}$ after 3 iterations (the left plots in Figure 7). The RMS of the mean strip residuals is equal to $6.53 \mathrm{~mm}$ before alignment and $0.945 \mathrm{~mm}$ after 3 iterations (the left plots in Figure 8). The larger final RMS for the strip mean residuals is, most likely, due to the misalignments of the strips within modules relative to the module centers. The geometry of strips within a module varies between the modules, and the correct placement of the strips in the module is unknown. To improve alignment of individual strips in a module, the data from the module mapper station should be used to determine the correct placement of the strips.

The right plots of Figures 7 and 8 show RMS of the individual module and strip residual distributions ${ }^{14}$. One entry is RMS of the residual histogram for a strip or a module, the examples of such histograms are shown in Figures 5 and 6 . There are no significant changes in these distributions with improved alignment. One possible explanation of this effect is rotational misalignment (Section 7). Such misalignments cause variations in the strip's transverse position depending on a location along the strip. The width of the module residual distribution also depends on the misalignment of the strips within the module.

In Figure 11 we plot the mean of the module mean residuals in a plane versus plane number.

\footnotetext{
${ }^{14}$ The different number of strips between iterations 2 and 3 is caused by the error in the tracking software when plane 120 was excluded. After the error was corrected, there are 96 more active strips in the data.
} 

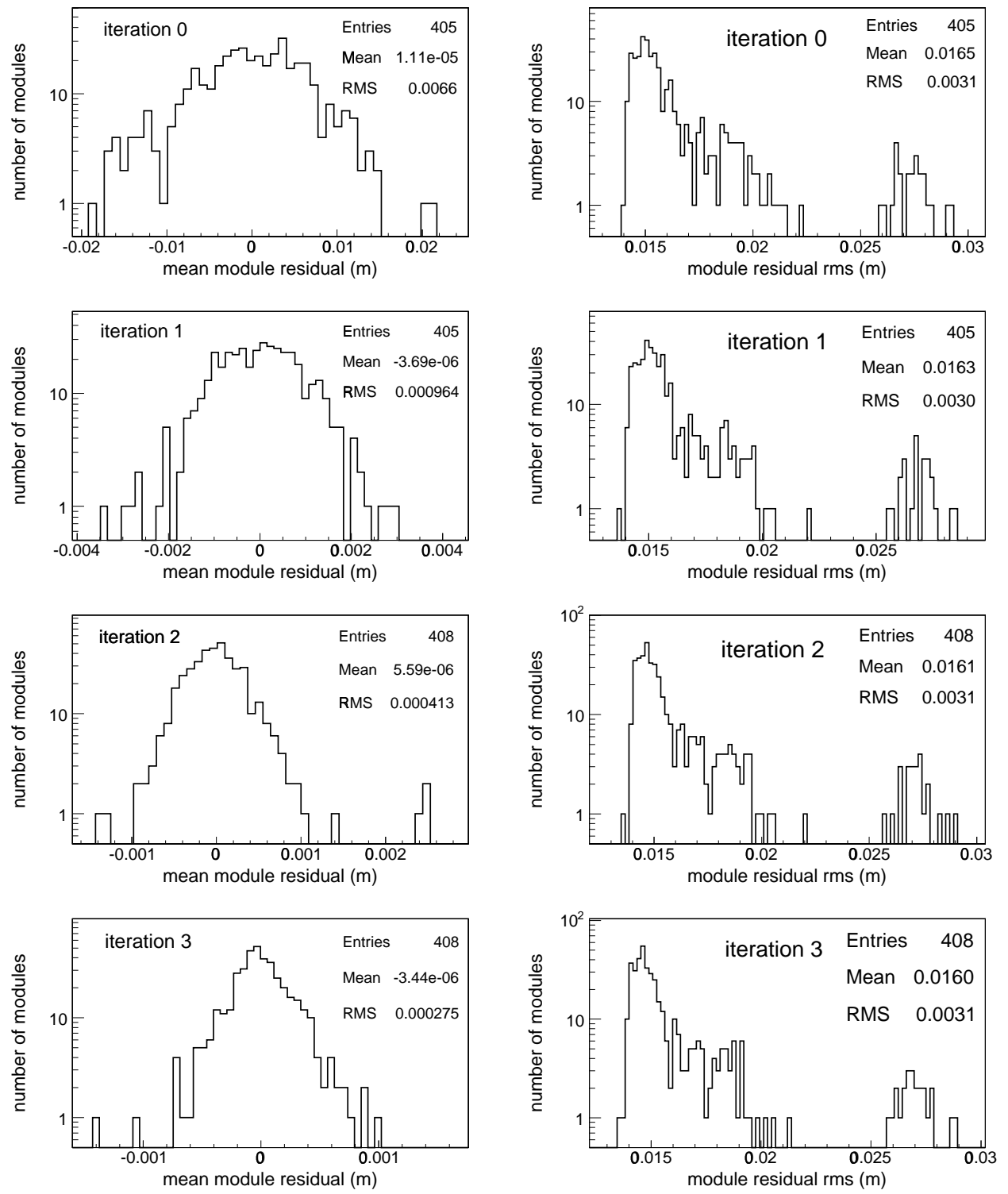

Figure 7: Mean and RMS of module residuals for 3 alignment iterations. These mean residuals are calculated using the muon tracks that pass the cuts. 

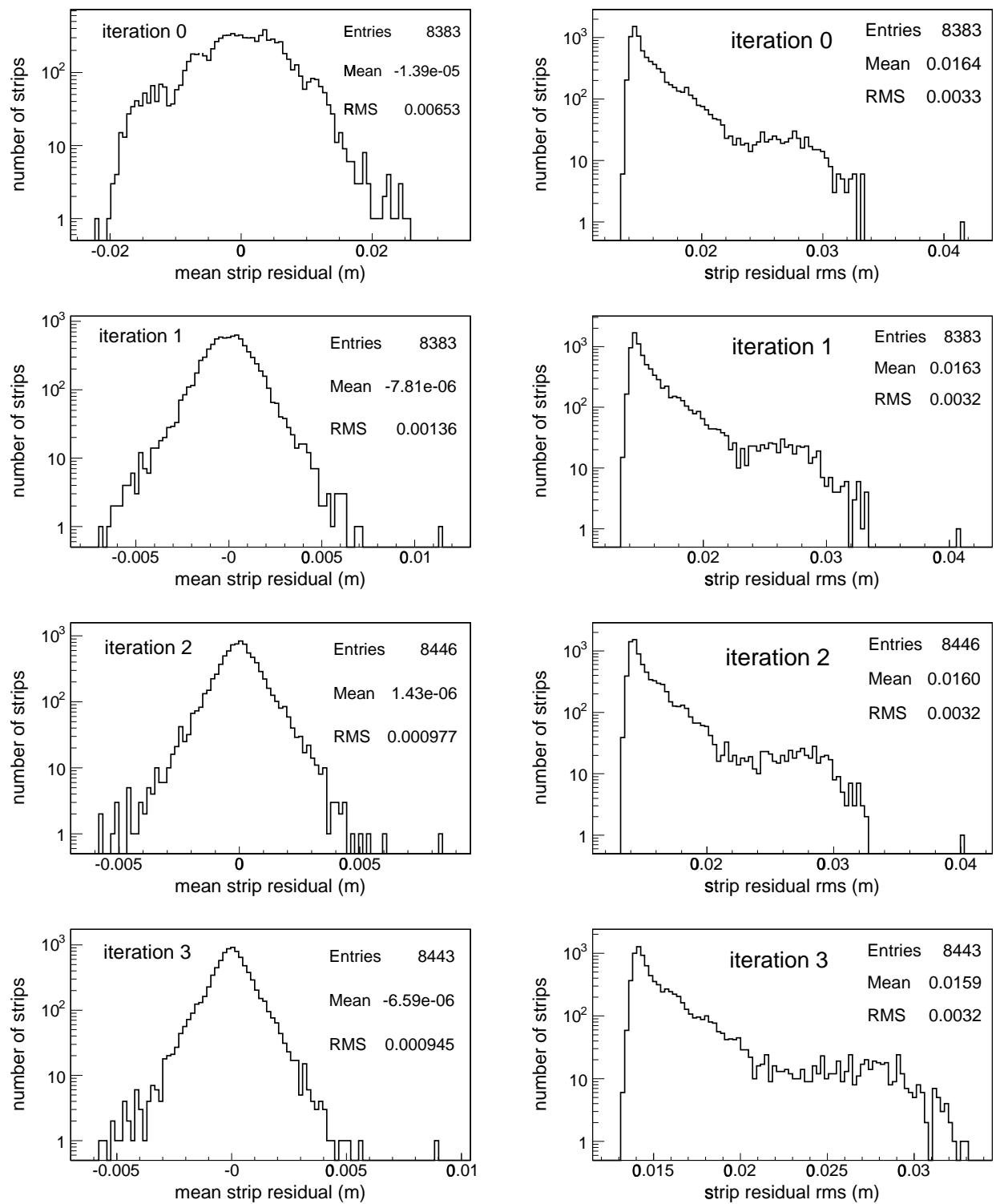

Figure 8: Mean and RMS of strip residuals for 3 alignment iterations. These mean residuals are calculated using the muon tracks that pass the cuts. 

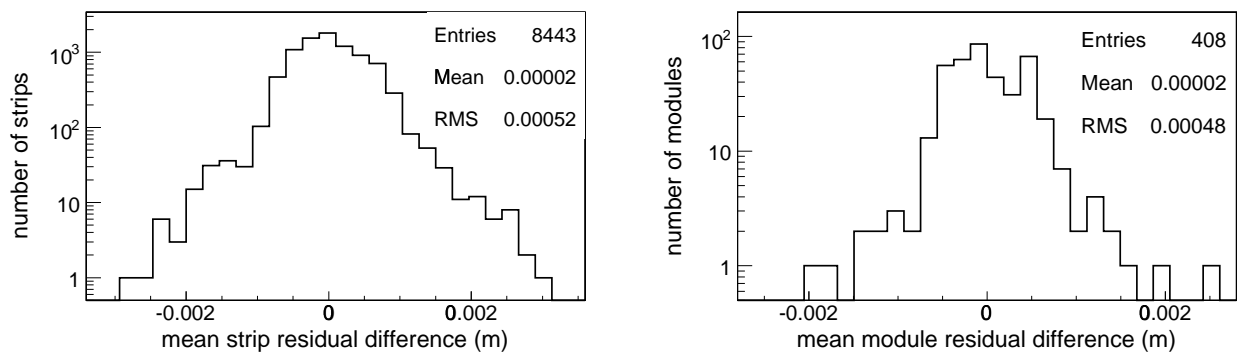

Figure 9: The difference between the mean strip and module residuals calculated with and without the track cuts, both calculations use the same improved geometry after the alignment. The residual differences are of the same size as the fluctuations in the calculations of the residuals described in Section 5 .

The errors bars are the standard deviation of the mean module residuals for the modules contained in the plane. As the detector alignment improves, the relative misalignments between the planes decrease from few millimeters to less than a millimeter. In Figure 10 we show four similar plots for the calorimeter strips.

\section{$5 \quad$ Alignment stability}

We would like to study the dependence of the alignment results on statistical fluctuations and systematic biases. We separate the alignment data set into 13 subsets. The subsets are ordered chronologically and each subset consists of approximately 1.3 million muon tracks. The data in the first 11 subsets span 414 hours and are collected before the magnet coil is energized for a first time. The last two subsets consist of total 49 hours of cosmic ray muons recorded with unknown residual magnetic field (current in the magnet coil is still off). In each subset, we calculate the mean strip and module residuals using all tracks in a subset that pass through a given strip or module. The variations in the mean residuals between subsets give estimates for the precision of the alignment procedures. For all calculations in this section we use the final detector geometry (after the iteration 3).

There is a shift of the detector mean residual in the last two bins in Figure 12. This shift is most likely caused by the residual magnetic field in the detector. The remaining strip and module misalignments, as shown in Figures 7 and 8, are on the order of $0.95 \mathrm{~mm}$ for strips and $0.28 \mathrm{~mm}$ for modules. Using Figure 12, an empirical estimate for the size of the magnetic field effect is around 0.05-0.1 $\mathrm{mm}$ and is much smaller than the required alignment precision.

We calculate the mean strip and module residuals using muon tracks from one subset. In the plots in Figure 13, one entry is the standard deviation over the 13 mean residuals, one mean residual for one subset. There is one entry per strip or module. The variations between the different subsets is on the order of $0.14 \mathrm{~mm}$ for strips and $0.6 \mathrm{~mm}$ for modules. These numbers suggest an existence of the lower statistical bound for the precision of the alignment calculations. Further studies will be required to understand these variations, in particular dependence of the standard deviation of mean residuals on different subset sizes.

\section{Comparison with the independent data set}

All three alignment iterations are done using the same set of cosmic ray muon data. To rule out a possible bias we use an independent data set to analyze the remaining module misalignments. The comparison data set consists of 16.7 million muon tracks. In Figure 14 we plot the mean strip and 

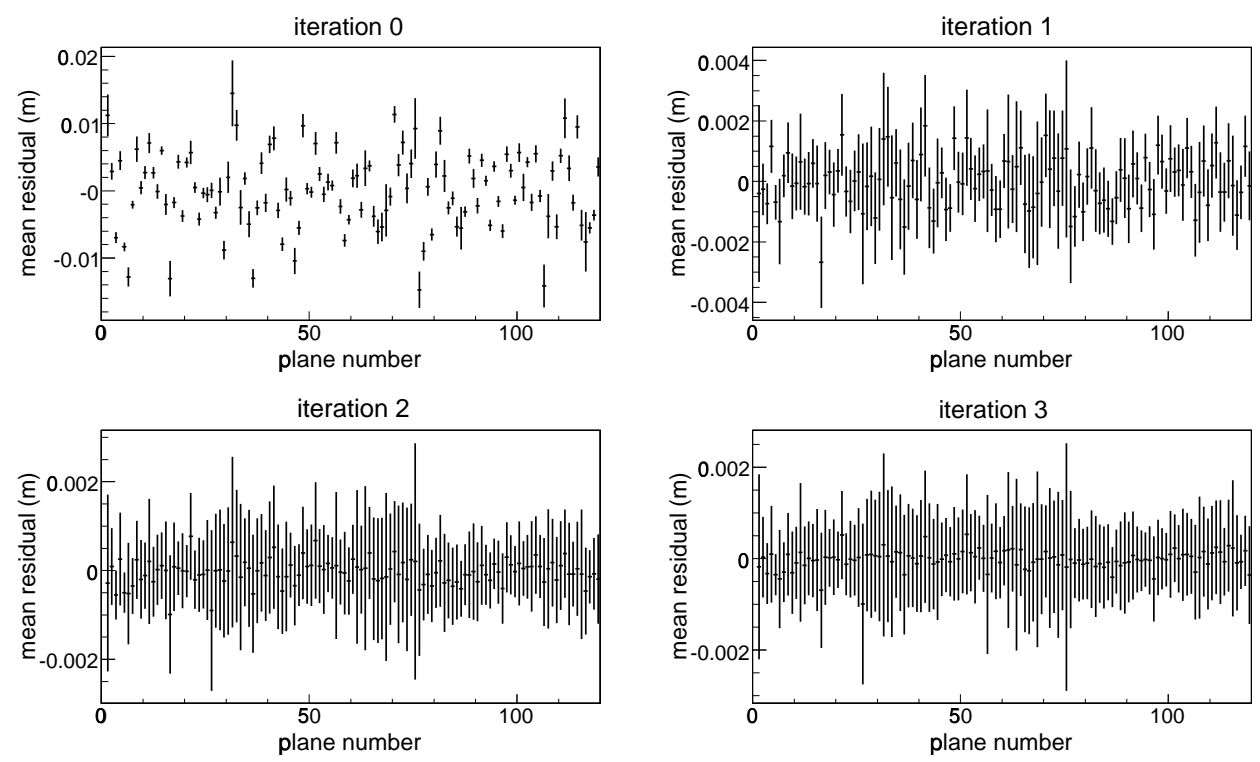

Figure 10: Mean of mean strip residuals in a plane versus plane number for 3 alignment iterations. Error bars are sigma of mean strip residuals over all strips in a plane.

module residuals calculated using the independent cosmic ray muon data. These calculations use the improved detector geometry after iteration 3 .

We calculate the mean strip and module residuals using two independent sets of the data. Then, for each calorimeter strip and module we calculate the difference between two mean residuals. In Figure 15 we plot these differences between the mean residuals calculated with the alignment data (after iteration 3) and the mean residuals calculated with the comparison data set. Both calculations use exactly the same detector geometry. The differences in the mean strip residuals are on the order of $0.2 \mathrm{~mm}$. The differences in mean module residuals are on the order of $0.1 \mathrm{~mm}$. Most of the data taken with the unmagnetized detector steel is already included in the alignment data set. The data in the comparison set are recorded in December, 2004 and January, 2005, after the magnet coil was energized. In Section 5 we show that the effect of the residual magnetic field on the alignment is smaller than $0.54 \mathrm{~mm}$ for the strips and $0.13 \mathrm{~mm}$ for the modules, which is consistent with the differences shown in the Figure 15. We conclude that there is a limit to the achievable precision of the alignment with the cosmic ray muons and using larger number of muon tracks does not further improve the alignment.

\section{Plane rotations}

The scintillator strips are placed in the vertical plane orthogonal to the Z-axis. Within a plane, all strips are expected to be parallel to either the U or V coordinate axis. Deviations between the longitudinal direction of a strip and the coordinate axis are termed rotational misalignments. In this section, we show that such misalignments are smaller order effects than the misalignments of the module transverse positions. We only estimate the magnitude of the rotational misalignments and do not correct them.

In order to calculate an angle between actual direction of a strip and the coordinate axis, we plot mean residual versus distance along the strip. Due to the low statistics, calculating the rotational offsets for individual strips does not produce consistent results. Instead, we plots residuals for all strips in a plane versus position along the coordinate axis orthogonal to the strips. A few examples are shown in Figure 16. The systematic variations in the residuals along the strips indicate rotational misalignments of the plane as a whole. 

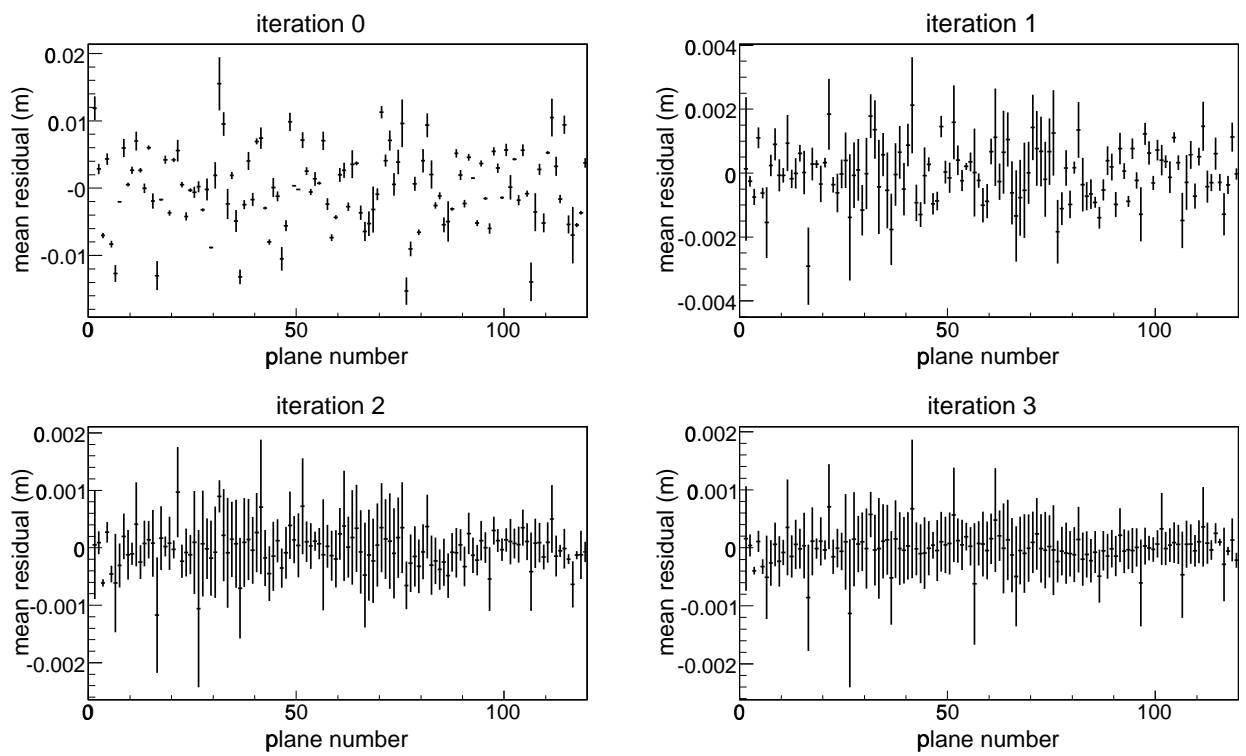

Figure 11: The mean of the mean module residuals in a plane versus plane number. The error bars are the sigmas of the mean module residuals over all modules in a plane. All 15.5 million muon tracks are used to make these plots.
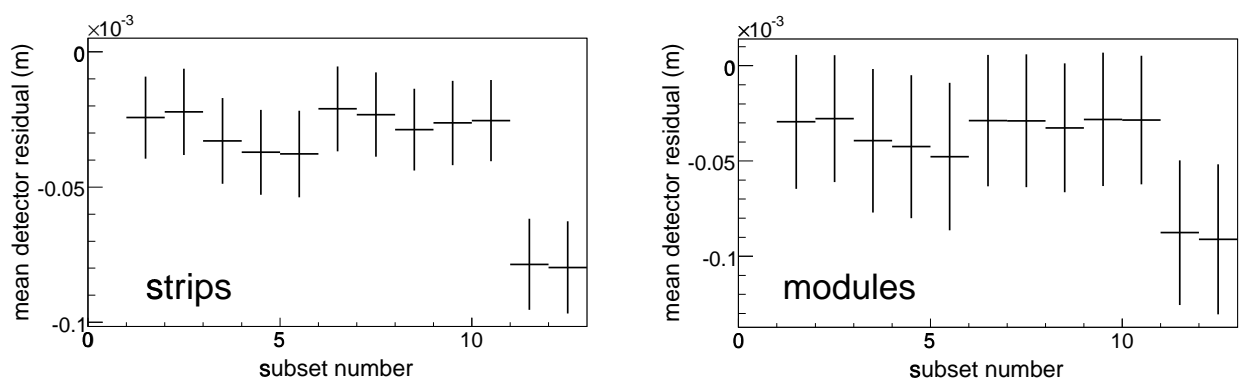

Figure 12: Mean of the mean strip and module residuals plotted versus subset number. For each bin, we average over mean residuals for all strips or modules in the calorimeter part of the detector. Error bars are spread over square root of number of strips or modules.
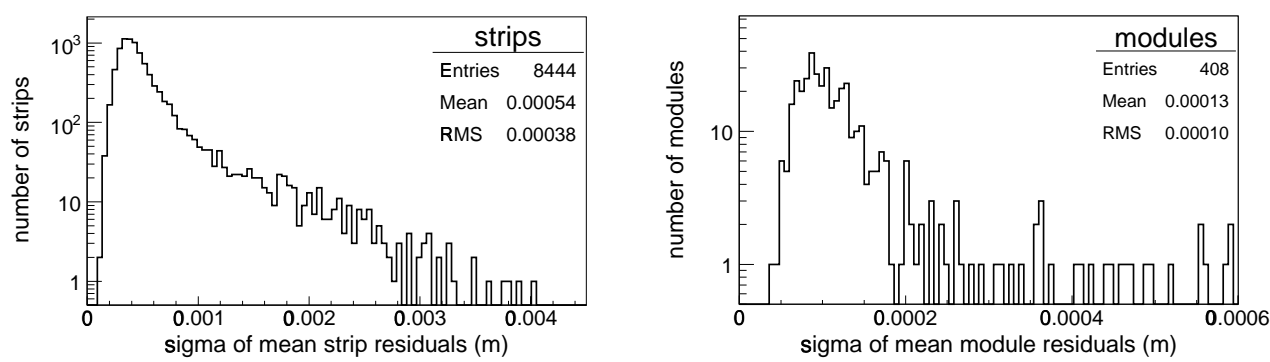

Figure 13: Sigma of the mean strip and module residuals calculated over 15 subsets. Sigma is the standard deviation of the 15 mean residuals for each strip and module. 

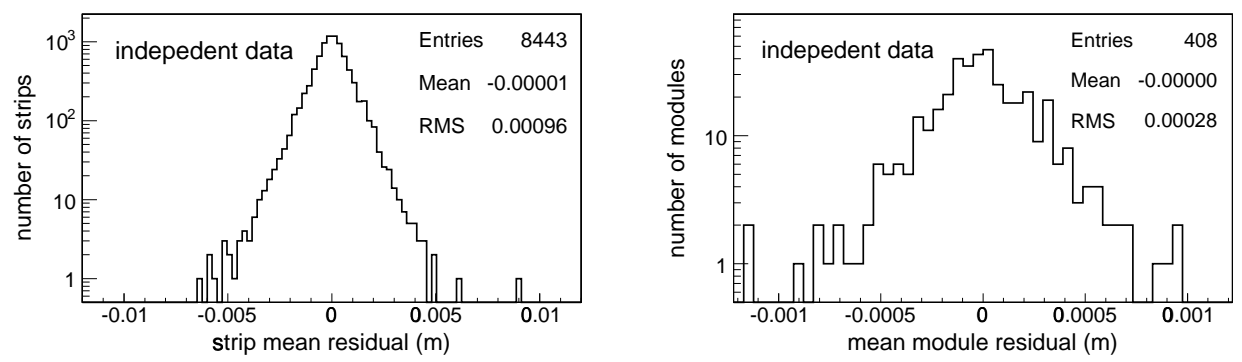

Figure 14: Mean strip and module residuals calculated using the independent data set of 16.7 million cosmic ray muon tracks.
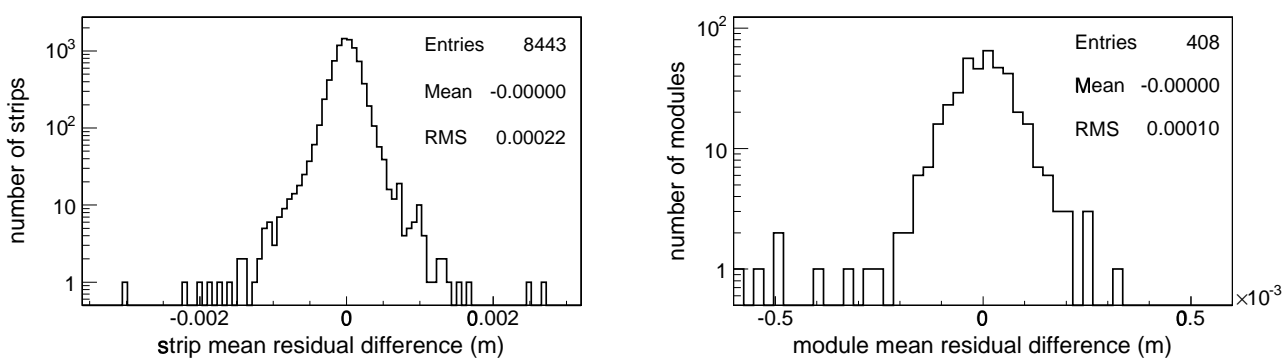

Figure 15: The difference between mean residual calculated with alignment data after iteration 3 and calculated with the comparison data. The left plot is for strips and right plot is for modules.

In order to determine the misalignment angle for each plane, we fit the plane rotational histogram (as in Figure 16) with a straight line. The slope of the best fit line gives the rotational misalignment angle. In Figure 17, we plot the angle of the best fit line before and after alignment for all calorimeter planes. There are no significant changes in the rotational offsets before and after the alignment (see Figure 18). This suggests that two effects are probably not correlated. There few planes that have misalignment angles on the order of 0.01 radians. Over $1 \mathrm{~m}$ strip length this corresponds to transverse misalignment of about $1 \mathrm{~cm}$. We conclude that for muon hits near strip's ends, the rotational misalignments become a significant effect for some calorimeter planes. Such misalignments have to be addressed in the future alignment work.

\section{Alignment consistency checks}

For each alignment iteration, the residuals are calculated based on the geometry from the previous iteration. In this section we compare the detector geometry before the alignment and after the completion of 3 alignment iterations. All plots in this section compare absolute geometries, without reference to the cosmic ray muon data. In this section we compare modules' absolute transverse positions before and after alignment.

In Figure 19, we plot the difference between the module transverse positions before and after alignment. These differences are consistent with the initial detector misalignment, as shown in Section 4. Figure 20 shows the same differences for four types of scintillator planes: Partial U, Full U, Partial V, Full V. There are small systematic differences in the module alignment corrections for different types of planes.

In order to check for the overlapping modules, we calculated the gaps between two neighboring modules. Figure 21 shows the difference between transverse positions of the edge strips in two neigh- 

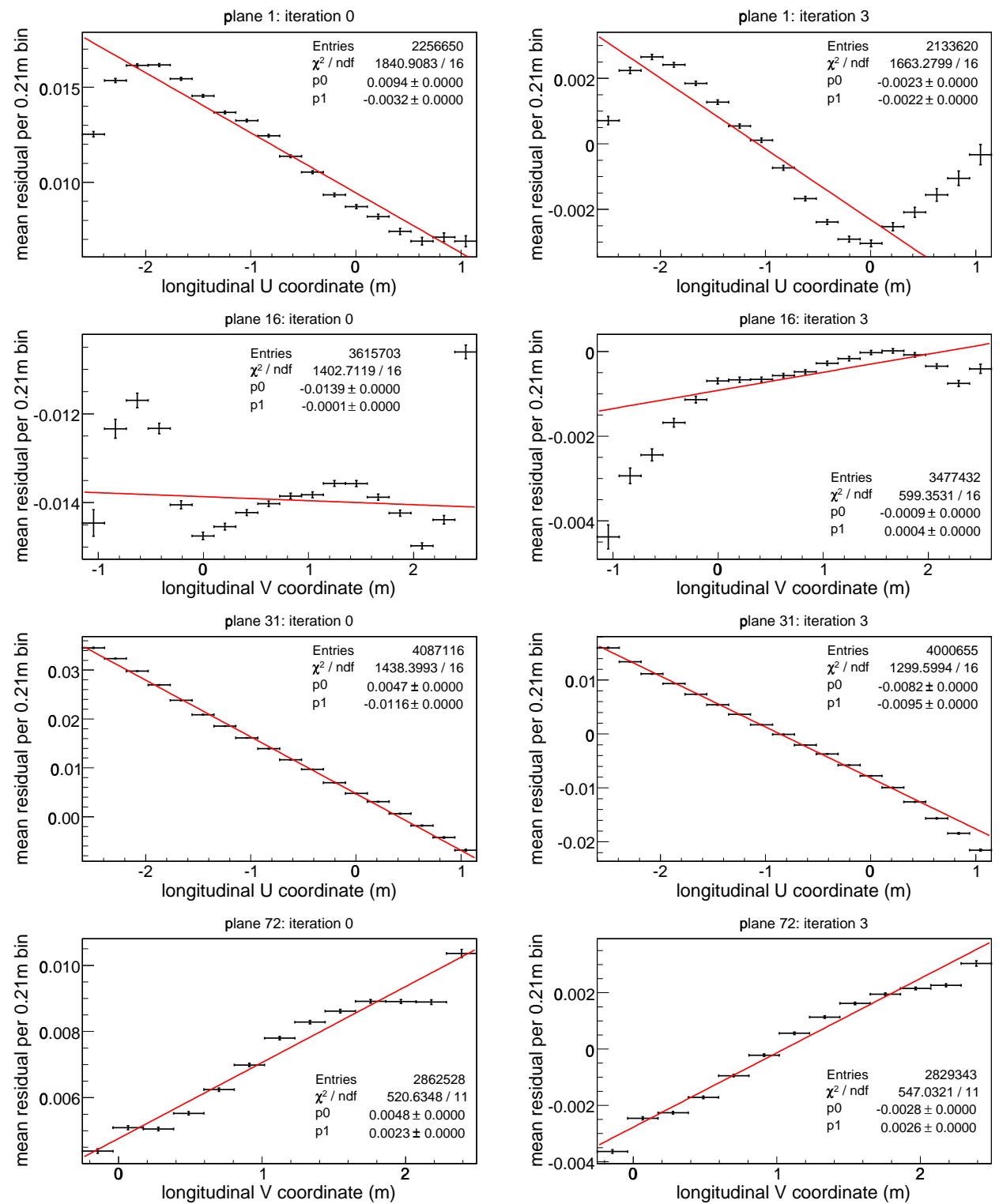

Figure 16: The examples of the plane rotations for four random calorimeter planes, before (left) and after (right) alignment. On Y-axis is the mean residual, where the mean is taken over all entries in one $\mathrm{X}$-axis bin and all strips in a plane. On $\mathrm{X}$-axis is the longitudinal position of a muon hit along a coordinate axis which is parallel to plane's strips. For U view strips the $\mathrm{V}$-axis is parallel to the strips and vice versa. 

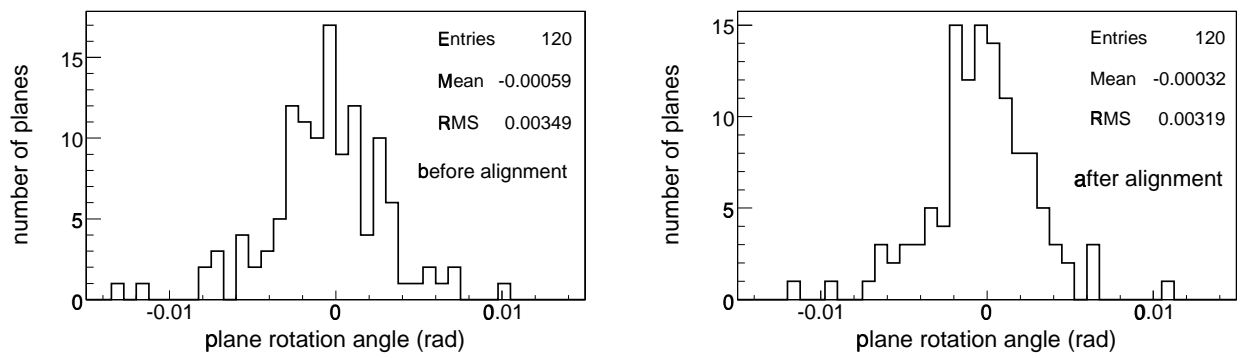

Figure 17: Plane rotation angle between best fit line along plane's strips and orthogonal U or V coordinate axis. Left plot is before and right plot is after this alignment is done.
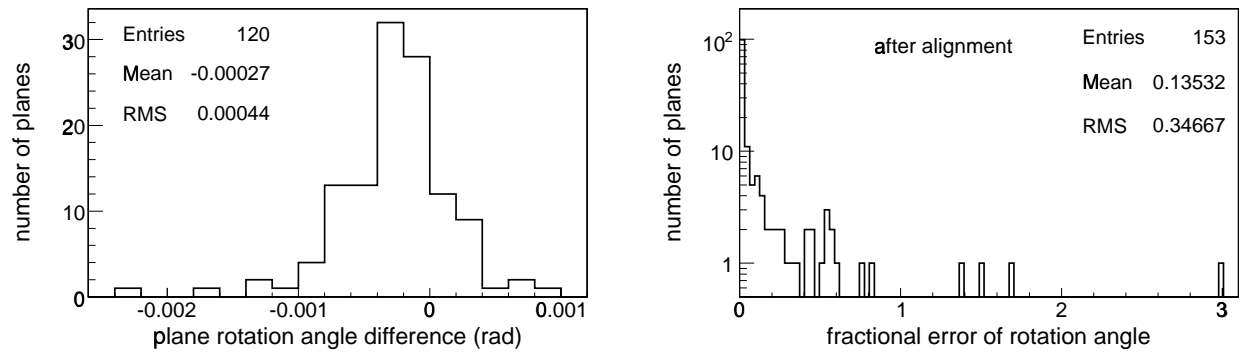

Figure 18: Left plot is difference in the rotation angles before and after the alignment. Right plot is fractional error of plane rotation angle after alignment: fit error over best fit value.
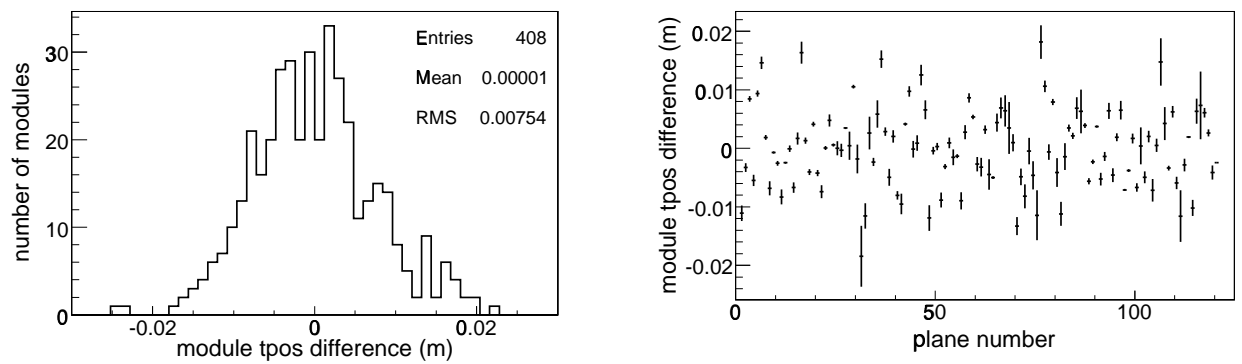

Figure 19: The left plot is the differences between the module transverse positions before and after alignment. In the plot on the right these differences are plotted versus plane number. The error bars are sigma over all modules in a plane. 

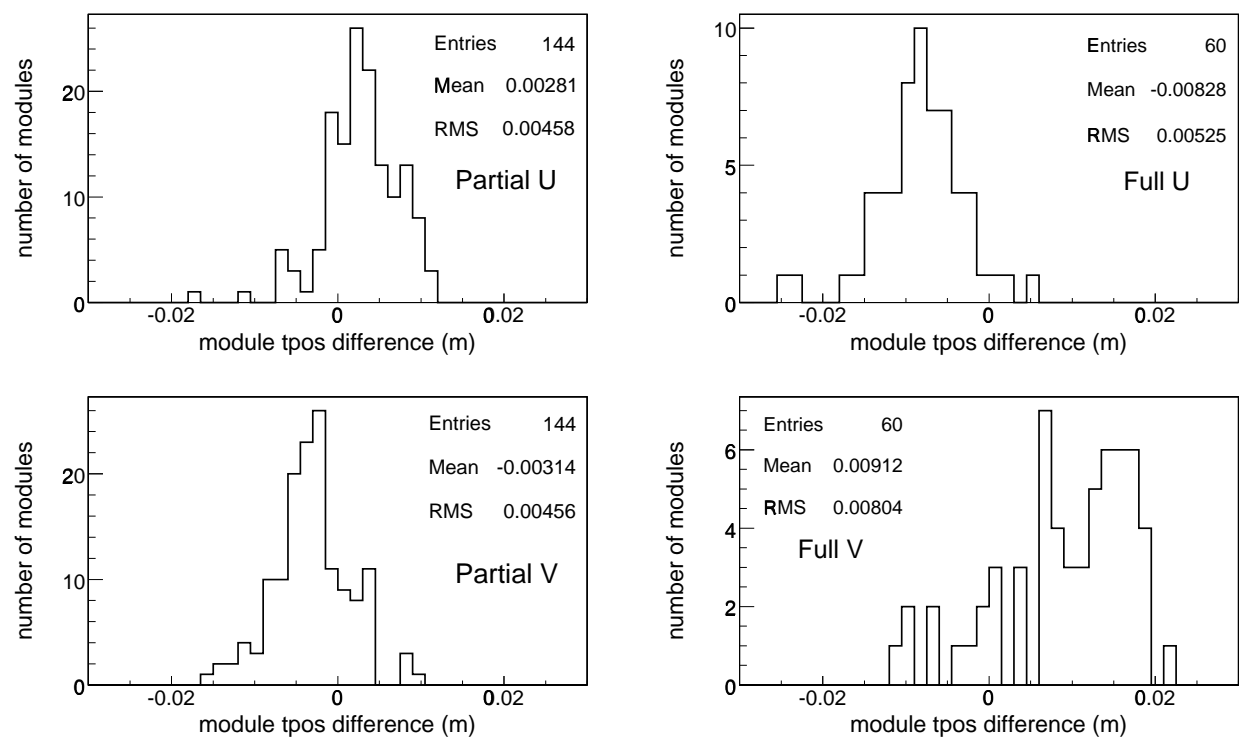

Figure 20: These plots show the differences between the module transverse positions before and after alignment for four types of scintillator planes.
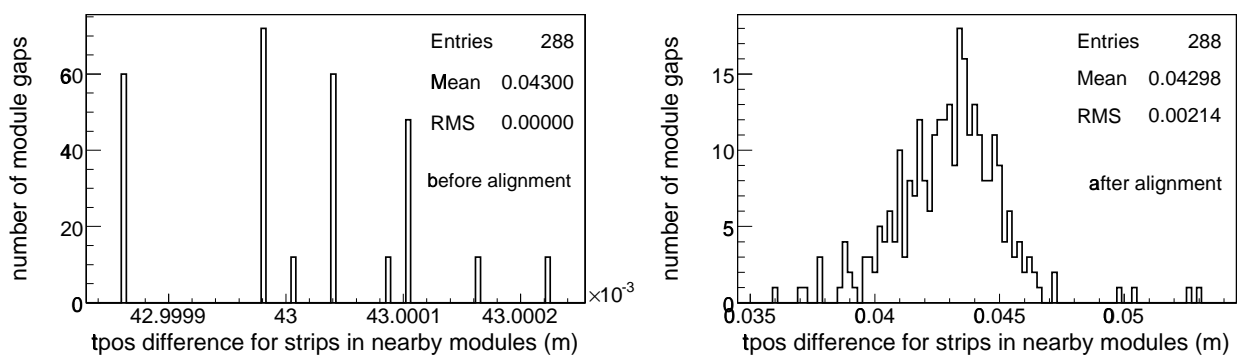

Figure 21: These plots show the gaps between neighboring scintillator modules. We plot the distance between the transverse positions of the edge strips in two neighboring modules. The distance which is less than a strip's width of $0.041 \mathrm{~m}$ corresponds to two overlapping modules. 
boring modules. Before alignment, the modules had discrete separations, which were larger than a strip width of $0.041 \mathrm{~m}$. After alignment, there are couple dozen modules that have overlapping edge strips. There are also a few module gaps larger than a centimeter. On average, the module gaps are close to the expected values.

\section{Conclusions}

Using 15.5 million muon tracks, we have aligned the scintillator strips in the calorimeter section of the Near Detector with better than $1 \mathrm{~mm}$ precision. This estimate is based on three facts. First, the statistical and systematic fluctuations in the mean residuals are on the order of $0.54 \mathrm{~mm}$ for the strips and $0.13 \mathrm{~mm}$ for the modules. Secondly, we compare the mean strip and module residuals, calculated using the independent set of 16.7 million muon tracks that are not used for the alignment, with the remaining residuals after the iteration 3 . These differences are on the order of $0.22 \mathrm{~mm}$ for the strips and $0.10 \mathrm{~mm}$ for the modules. Finally, the remaining residuals after the completion of the iteration 3 are on the order of $1.0 \mathrm{~mm}$ for the strips and $0.3 \mathrm{~mm}$ for the modules.

There are three main issues that still need to be addressed:

- Alignment of the spectrometer

- Strip by strip alignment

- Plane rotational alignment

Each of these issues pose certain challenges and require concentrated efforts in order to resolve them.

Spectrometer alignment is complicated by the inconsistencies in track finding for muon hits in the spectrometer strips ${ }^{15}$. An improvement in the track finding algorithm is required in order to be able to proceed with the spectrometer alignment. The alignment of the strips within the modules should include mapper test stand data from the Argonne National Laboratory, in addition to the cosmic ray muon data.

Finally, before the rotational misalignments are corrected, the track finding algorithm has to be modified. These modifications have to account for the varying transverse position of a muon hit along a strip length. Currently, when forming $2 \mathrm{~d}$ tracks, the track finder uses transverse strip position at the geometrical center of the strip. For the planes that have few miliradians angular misalignments, the track finder has to be modified to use the transverse position at the location of the muon hit.

\section{Acknowledgments}

We would like to thank many MINOS collaborators who helped us with this work. In particular, we relied on Bernard Becker, David Boehnlein and Brett Viren for the details of the Far Detector alignment. Brett Viren also provided frequent and valuable help with developing the alignment code. We would like to thank Robert Hatcher for the useful explanations of the Near Detector geometry simulation and for being our main reference for the MINOS offline software. Frequent discussions with Peter Shanahan helped us to understand better the alignment issues and ensured the timely completion of this work. We also would like to thank Elizabeth Barnes for helping with the final draft.

\footnotetext{
${ }^{15}$ These observations are based on the results obtained with version R1.13 of the MINOS reconstruction software.
} 


\section{Appendix A Dependence of alignment on defective channels}

Muons in the Near Detector produce scintillation light in the strips that they cross. Photomultipliers convert the light to the electric charge and the charge is digitized by the front end electronics. Reconstruction software forms tracks using strips that have non zero charge. In addition to channels with non zero charge caused by direct muon hits, there are other channels that have non zero charge output. Some of such channels are caused by photomultiplier and electronics crosstalk, noise hits and defective electronics channels ${ }^{16}$. Because our data were collected during the detector commissioning, there are changes in the Near Detector hardware that might affect the alignment results. We would like to estimate the variations in the residuals produced by the defective detector channels.
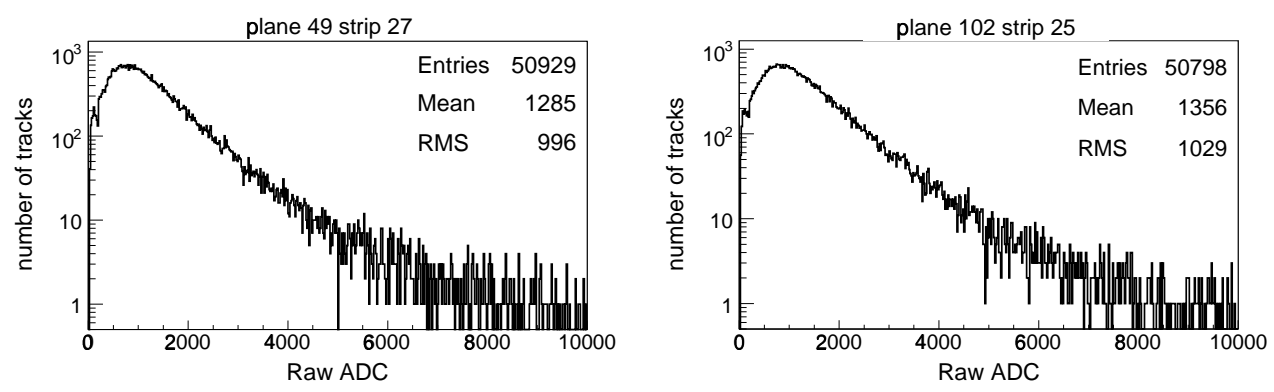

Figure 22: Two examples of charge distributions in normal channels when strips are on a track.
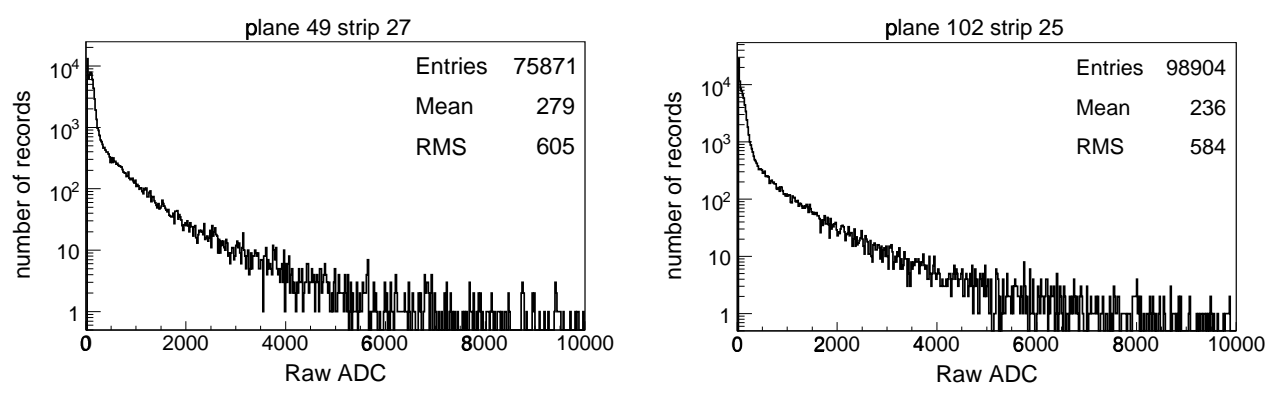

Figure 23: Two examples of charge distributions in normal channels when strips are not on a track.

For each record ${ }^{17}$, we separate the strips with non zero charge into two categories. First, we identify all strips that belong to a reconstructed muon track, as assigned by the track finding algorithm. We say that these strips are on track. All other strips in a record are then said to be off track or not on a track. For each physical strip in the detector we fill two histograms. One is filled with strip's charge when the strip is on track. The other is filled with strip's charge when the strip is off track. In Figures 22 and 23 we show two examples of histograms of each type. We are using 15.5 million records to fill these histograms ${ }^{18}$. In Figures 28 and 29 we plot the mean charge and the number of entries in these two types of histograms for each strip in the calorimeter part of the detector.

There is approximately a dozen of channels that produce a large number of off track entries. These channels are identified by visually scanning the off track charge histograms that have top $1 \%$ of the number of entries (the bottom left plot in Figure 29). Defective channels can be separated into three categories:

\footnotetext{
${ }^{16}$ We use term defective to identify channels with characteristics that are significantly different from the most channels in the detector.

${ }^{17}$ One record (or snarl) is one detector trigger event.

${ }^{18}$ We only select records that have exactly one reconstructed muon track.
} 

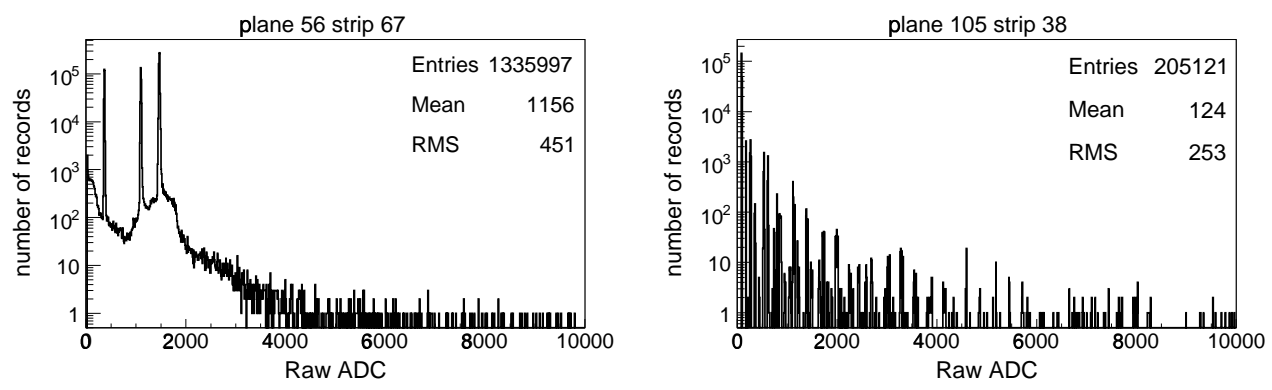

Figure 24: Two examples (out of total 3 strips) of distorted charge distributions in the defective strips when the strips are not on a track.
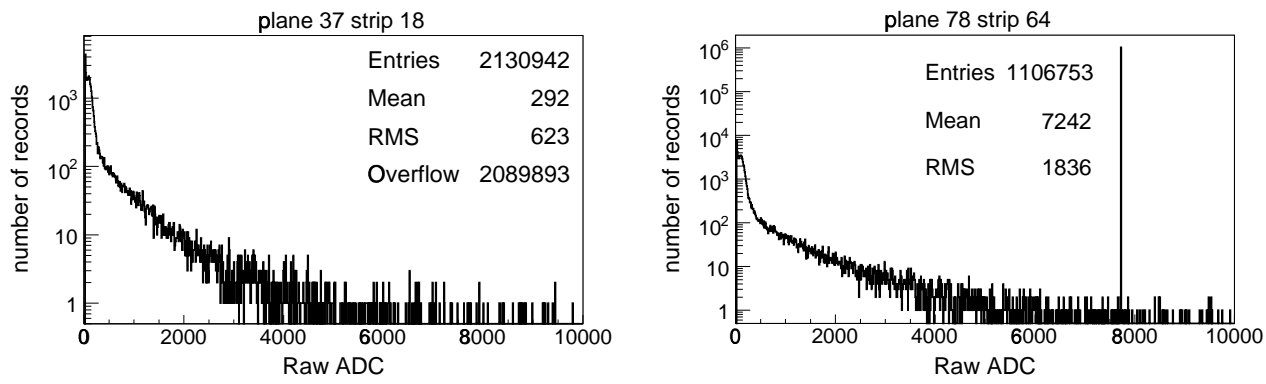

Figure 25: Two examples (out of total 8 strips) of abnormal charge distributions above 7000 ADC in the defective strips when the strips are not on a track.
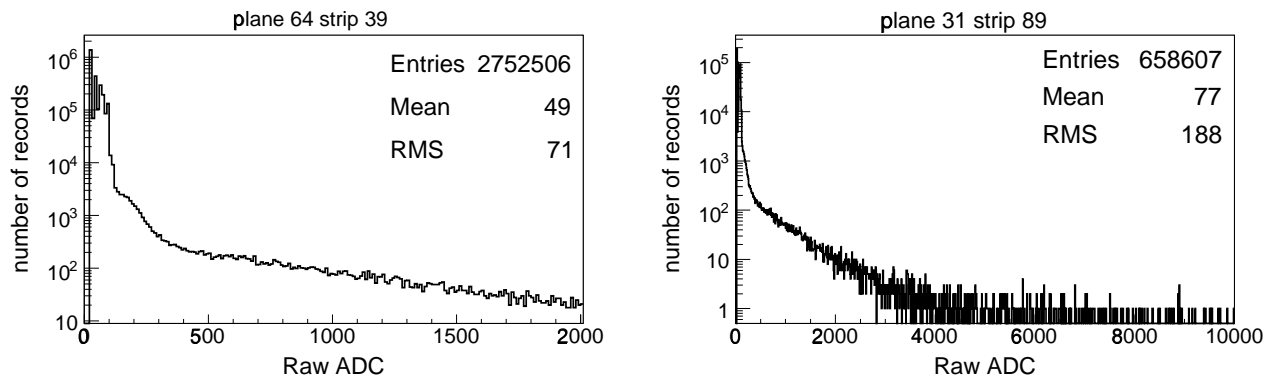

Figure 26: Two examples (out of total 7 strips) of abnormal charge distributions below 200 ADC in the defective strips when the strips are not on a track.
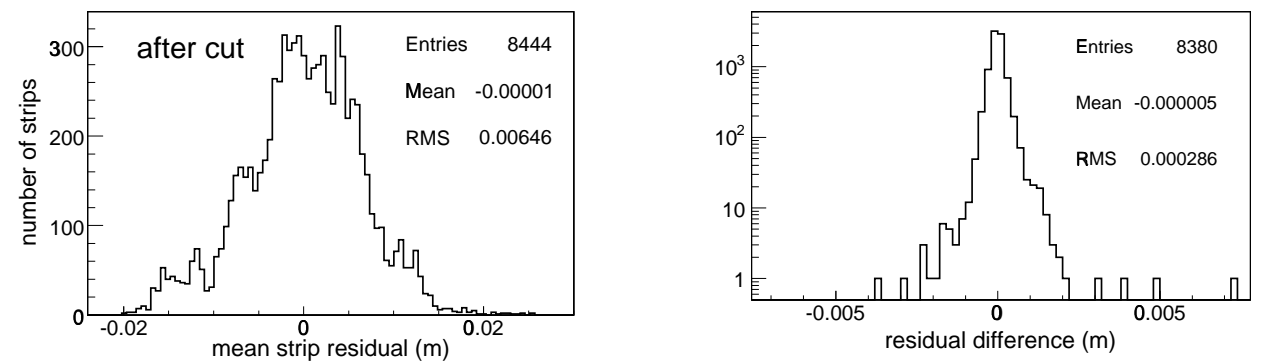

Figure 27: The left plot is the mean strip residuals of the calorimeter strips after the cut on defective channels. The right plot is the difference in the mean strip residual before and after the cut. All residuals are calculated using the initial detector geometry before the alignment. 
- Channels that have discrete or distorted off track charge distributions (Figure 24)

- Channels that have high number of entries with high ADC values (Figure 25).

- Channels that have high number of entries with low ADC values (Figure 26).

For comparison, Figure 23 shows two examples of normal charge distributions for strips that are not on a track. Strips with large off track charge output pull reconstructed muon tracks, acting as pivot points, and introduce systematic biases to the mean residual offsets for nearby strips. We remove the defective channels using these cuts:

- 3 channels are turned off due to anomalous charge distributions (Figure 24).

- 8 channels are cut above 7000 ADC (Figure 25).

- 7 channels are cut below 200 ADC (Figure 26).

In addition, we also suppress all digits ${ }^{19}$ that have charge less than $50 \mathrm{ADC}$ (this cut is roughly equivalent to 0.5 pe cut).

All of the alignment data are then reprocessed with these four cuts, using the initial geometry before the the alignment. In the left plot of Figure 27 we plot the mean strip residuals which are calculated using the four cuts above. In the right plot of Figure 27 we plot the difference between the mean strip residuals that are calculated with and without the four cuts. The RMS of this plot is equal to $0.3 \mathrm{~mm}$ and gives an estimate of the errors in calculating the mean strip residuals when there are defective channels in the data.
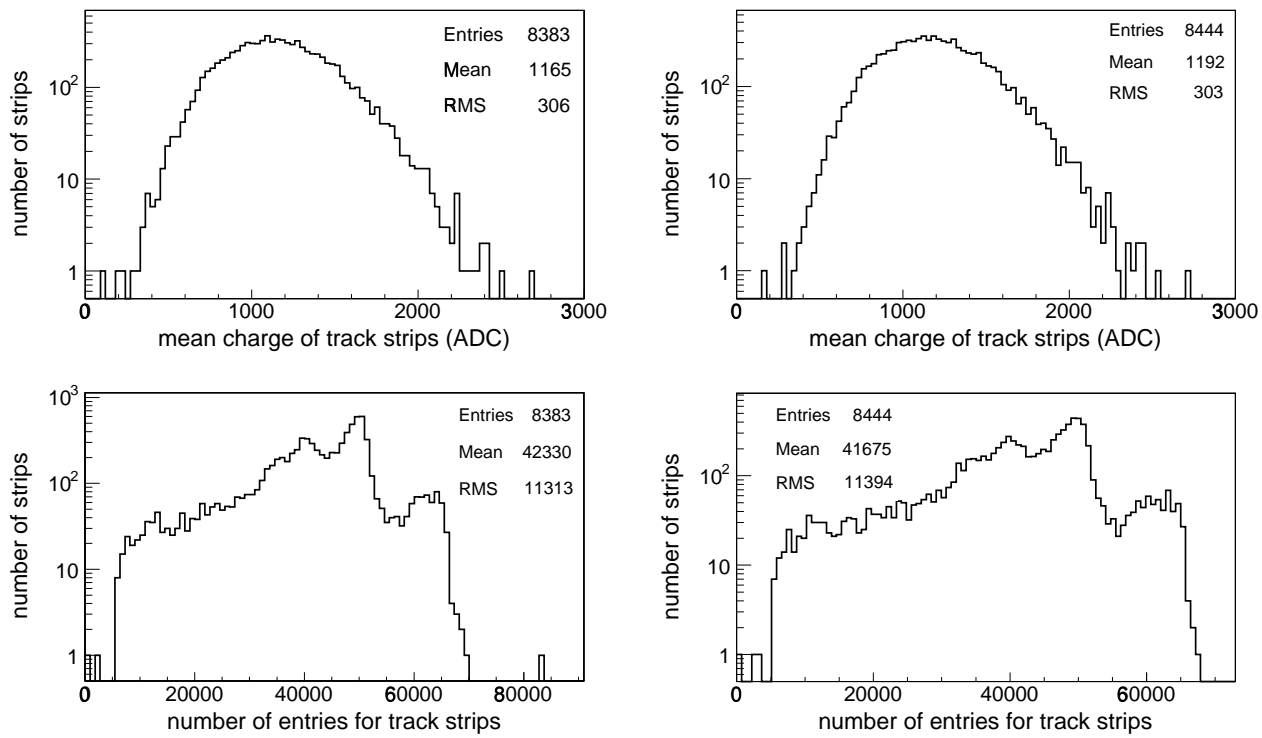

Figure 28: Mean charge and number of entries for strips that are on track. The plots on the left are before and the plots on the right are after the cut on defective channels.

In Figure 30 we plot a ratio of the reconstructed muon track charge over the sum of all charge in a record. After few defective channels are removed, muon tracks carry larger fraction of the charge recorded by the detector.

\footnotetext{
${ }^{19} \mathrm{~A}$ digit is a charge digitized over $18.7 \mathrm{~ns}$ time period.
} 

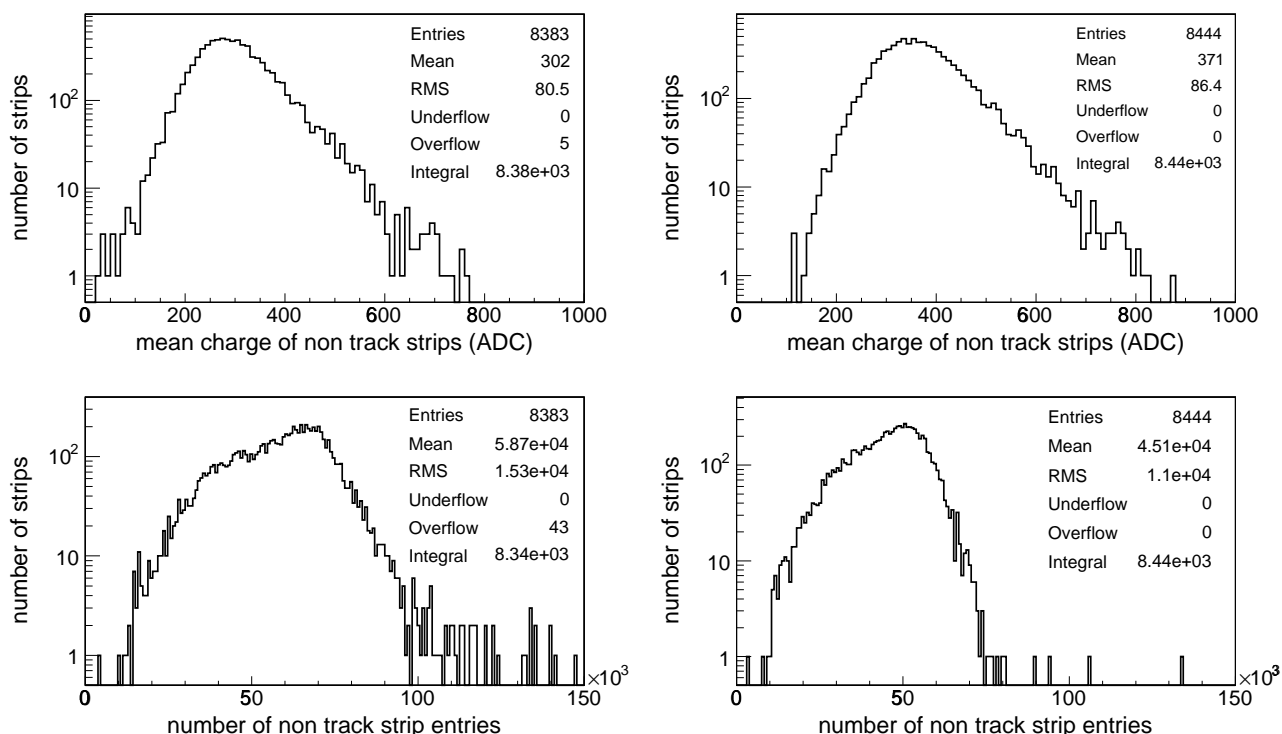

Figure 29: Mean charge and number of entries for strips that are not on track. The plots on the left are before and the plots on the right are after the cut on defective channels.
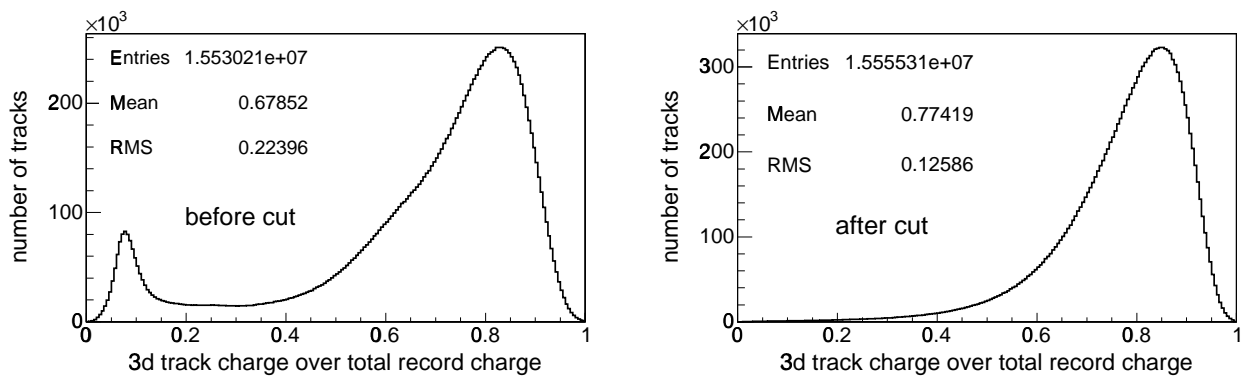

Figure 30: Ratio of muon track charge over total charge recorded in a record, before and after the cut on defective channels. After the cut more of the record charge is reconstructed as being on a track.

We chronologically separate 463 hours of the data into 46 equal size bins. For each bin, we calculate the mean of four quantities: number of strips on track in a record, charge of all strips on track in a record, number of strips off track in a record and charge of all strips off track in a record. In Figures 31, 33,32 and 34 we plot these four quantities versus time. There are significant variations in these four quantities between different time periods. These variations can not be be explained by the statistical fluctuations alone and, at least in part, are caused by the defective channels. We have shown in Section 6 that the such variations in the data do not introduce the systematic biases to the calculation of the mean strip and module residuals.

\section{Appendix B Dependence of track reconstruction on alignment}

The track finding algorithm uses strip positions for muon track reconstruction, so changes in the detector geometry influence reconstructed track quantities. In Figure 35, we plot the number of strips in the $\mathrm{V}$ and $\mathrm{U}$ views $2 \mathrm{~d}$ tracks for 3 alignment iterations. There are fewer strips reconstructed for $\mathrm{U}$ view $2 \mathrm{~d}$ tracks than $\mathrm{V}$ view after the alignment. The number of strips in the $\mathrm{V}$ view tracks has not 

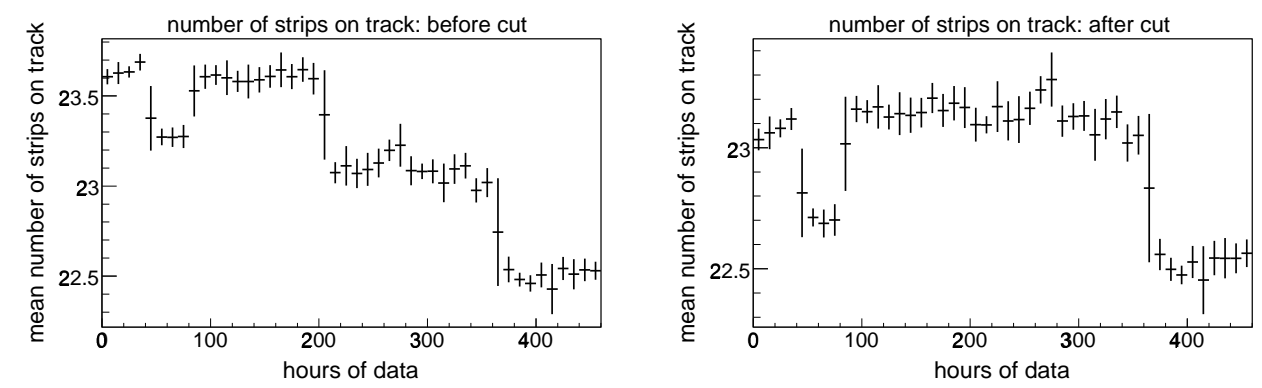

Figure 31: Mean number of strips belonging to muon tracks plotted versus time. The bins are 10 hours wide.
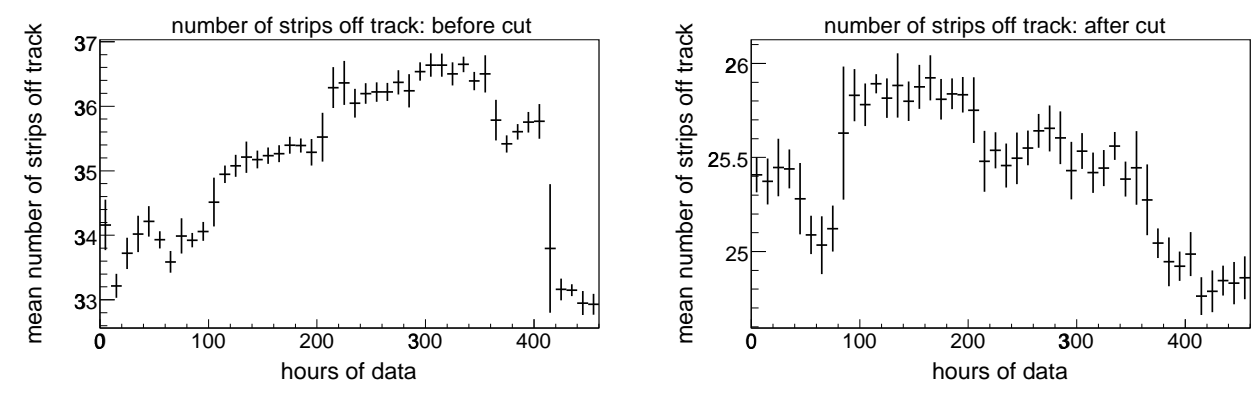

Figure 32: Mean number of strips in a record, with charges above sparsification threshold but which are not reconstructed as a part of a muon track, plotted versus time. The bins are 10 hours wide.
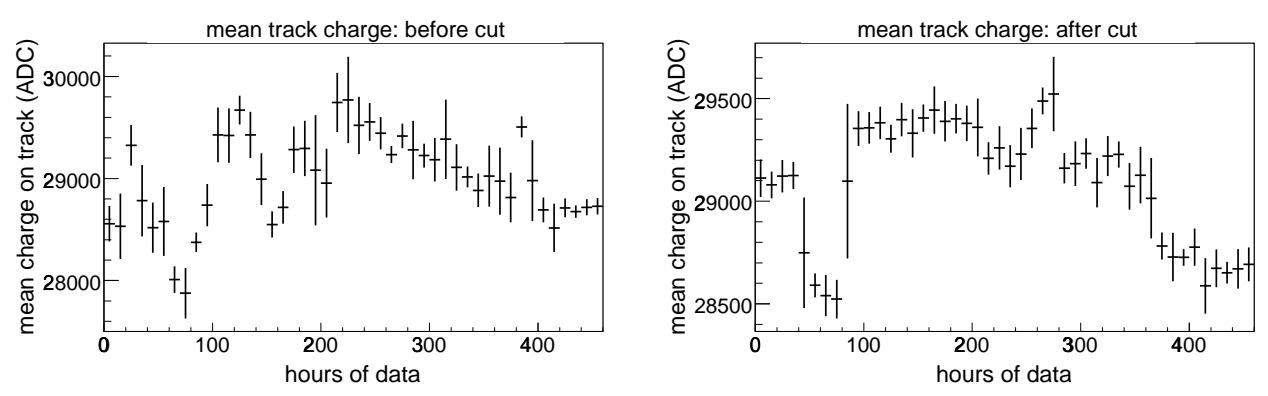

Figure 33: Mean muon track charge plotted versus time. The bins are 10 hours wide.
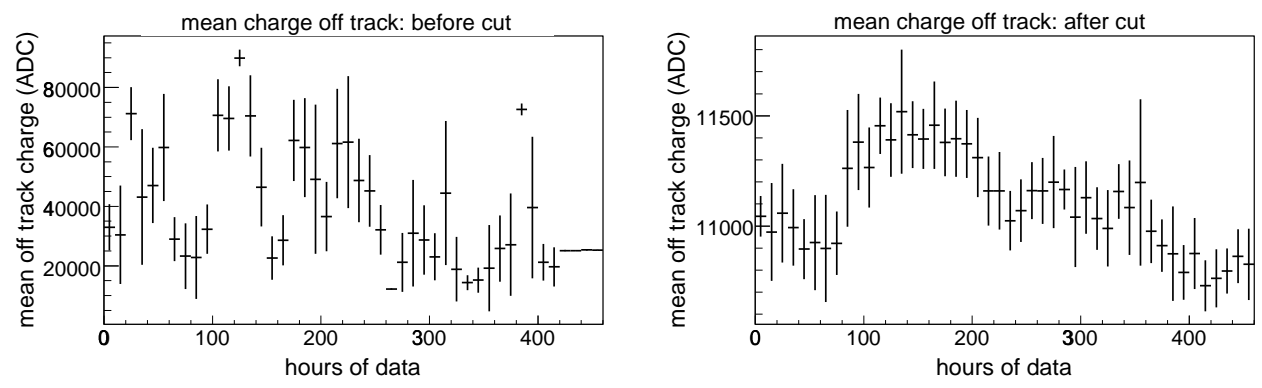

Figure 34: Mean charge of all strips in a record, which are not reconstructed as a part of a muon track, plotted versus time. The bins are 10 hours wide. 
changed much with improved alignment. In Figure 35 plots, the sharp decrease in number of strips around 5 is due to the trigger condition requiring simultaneous hits in multiple planes.

Changes in raw $2 \mathrm{~d}$ track charge are shown in Figure 36 . While there are fewer strips on $2 \mathrm{~d}$ tracks after the alignment, total $2 \mathrm{~d}$ track raw charge increases with the improved alignment. One possible explanation is due to the tracking algorithm selecting fewer strips as being on a track. But the new strips that are now included as part of a track, because they are better aligned with track direction, have larger amount of charge deposited by a muon.

For each $2 \mathrm{~d}$ track, we calculate the least squares sum $S$ using all $n$ fit points and the best fit parameters. Then, $\sigma=\sqrt{S /(n-2)}$ gives an estimate for the size of variations of the strip positions around the best fit line ${ }^{20}$. The size of $\sigma$ also gives an idea about the quality of the fit. In Figure 37 we plot $\sigma$ for 15.5 million muon tracks in the alignment data set. For iteration 0 , the peaks at $0.0025 \mathrm{~m}$ are caused by the tracks that pass through the strips with the same strip number, from one plane to the next. Misalignments introduce random shifts in the strip positions, hence breaking this degeneracy.

\footnotetext{
${ }^{20}$ In cases when uncertainty in the fit points is known, $\sigma^{2}$ is proportional to $\chi^{2}$.
} 

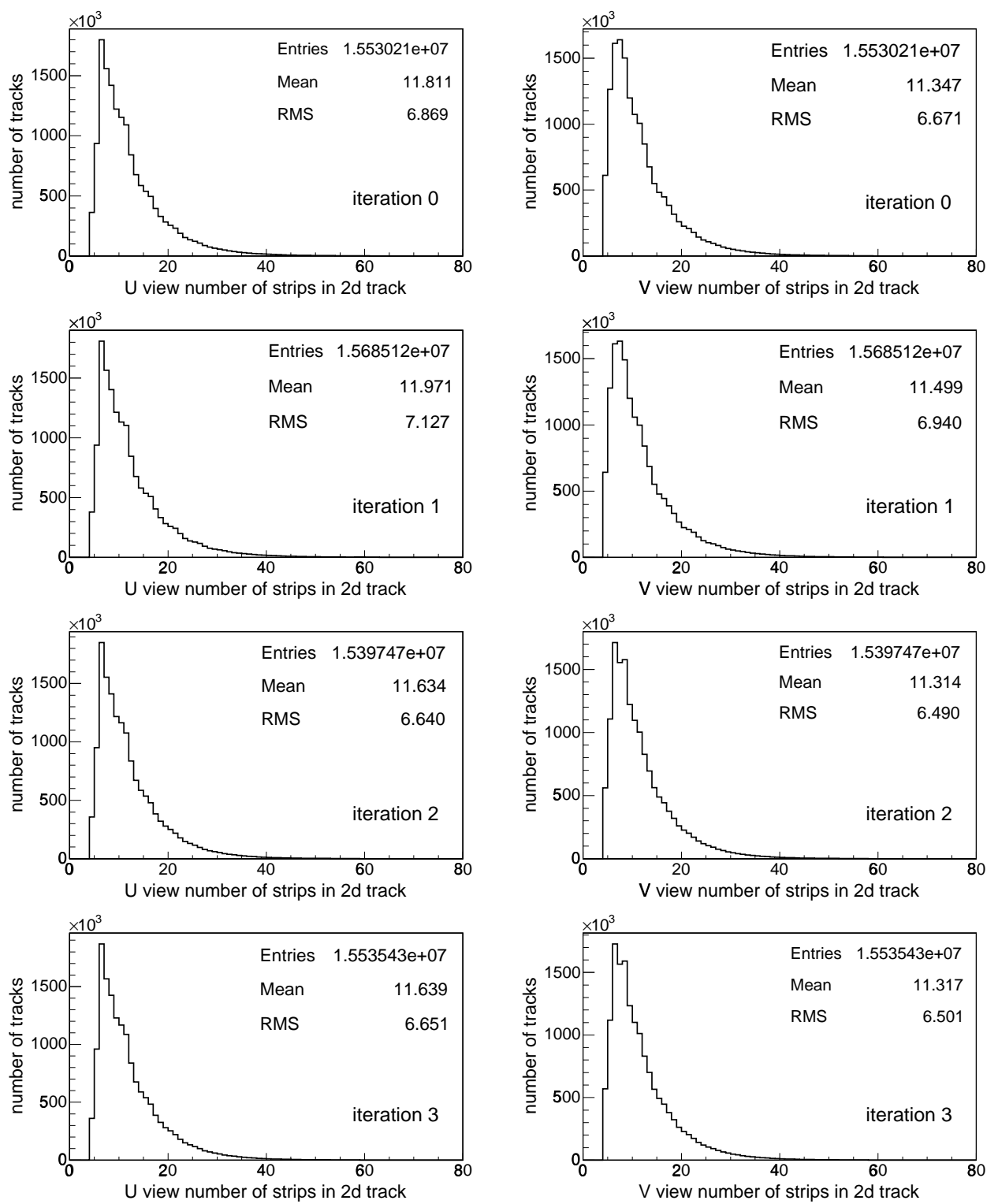

Figure 35: Number of strips in $2 \mathrm{~d}$ tracks for 3 iterations. 

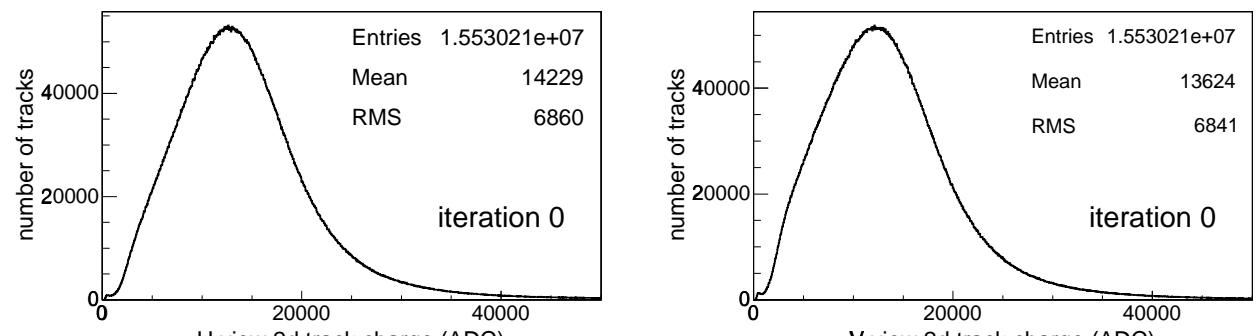

$\mathrm{U}$ view $2 \mathrm{~d}$ track charge (ADC)
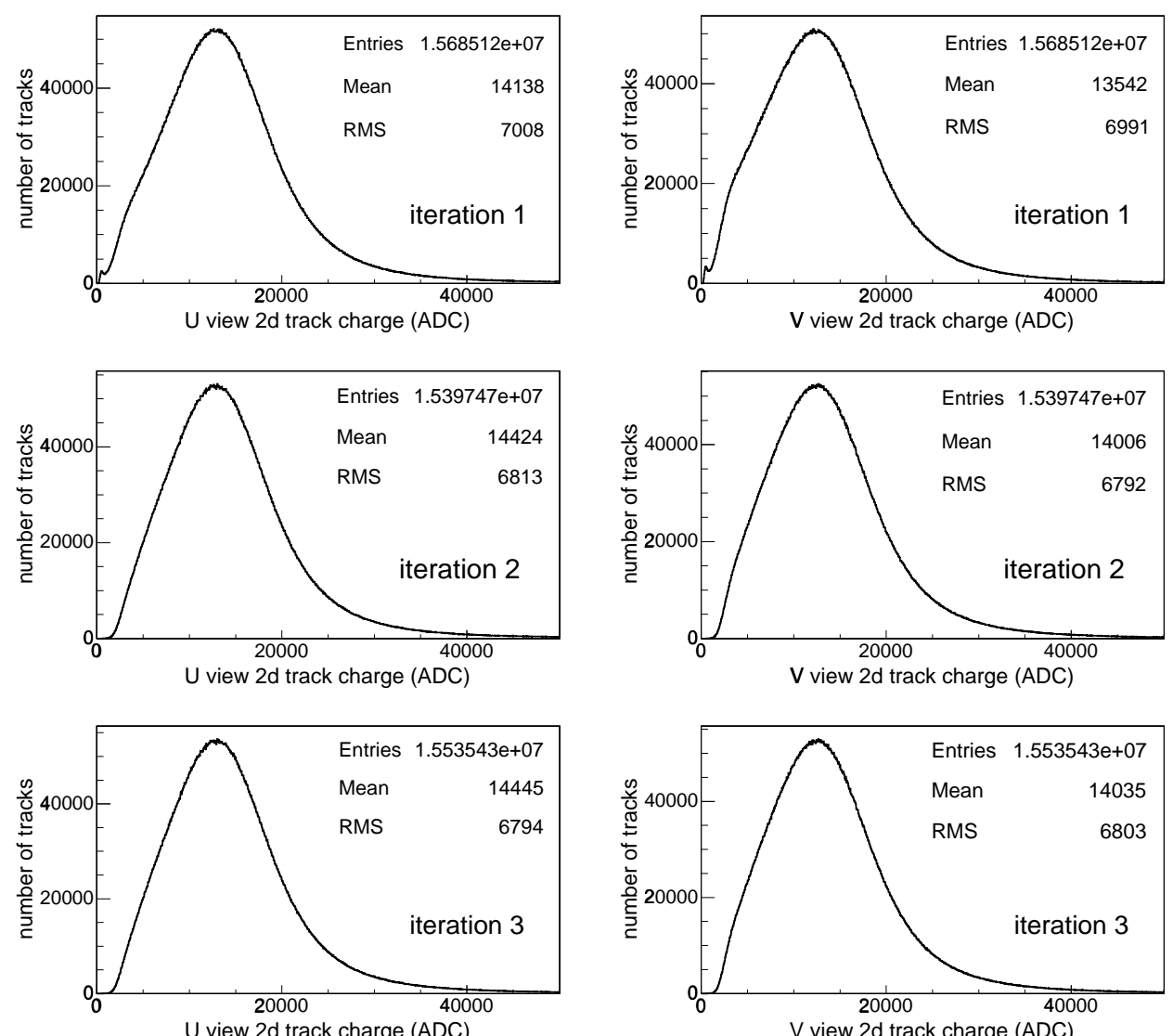

Figure 36: Charge of $2 \mathrm{~d}$ tracks for 3 alignment iterations. 

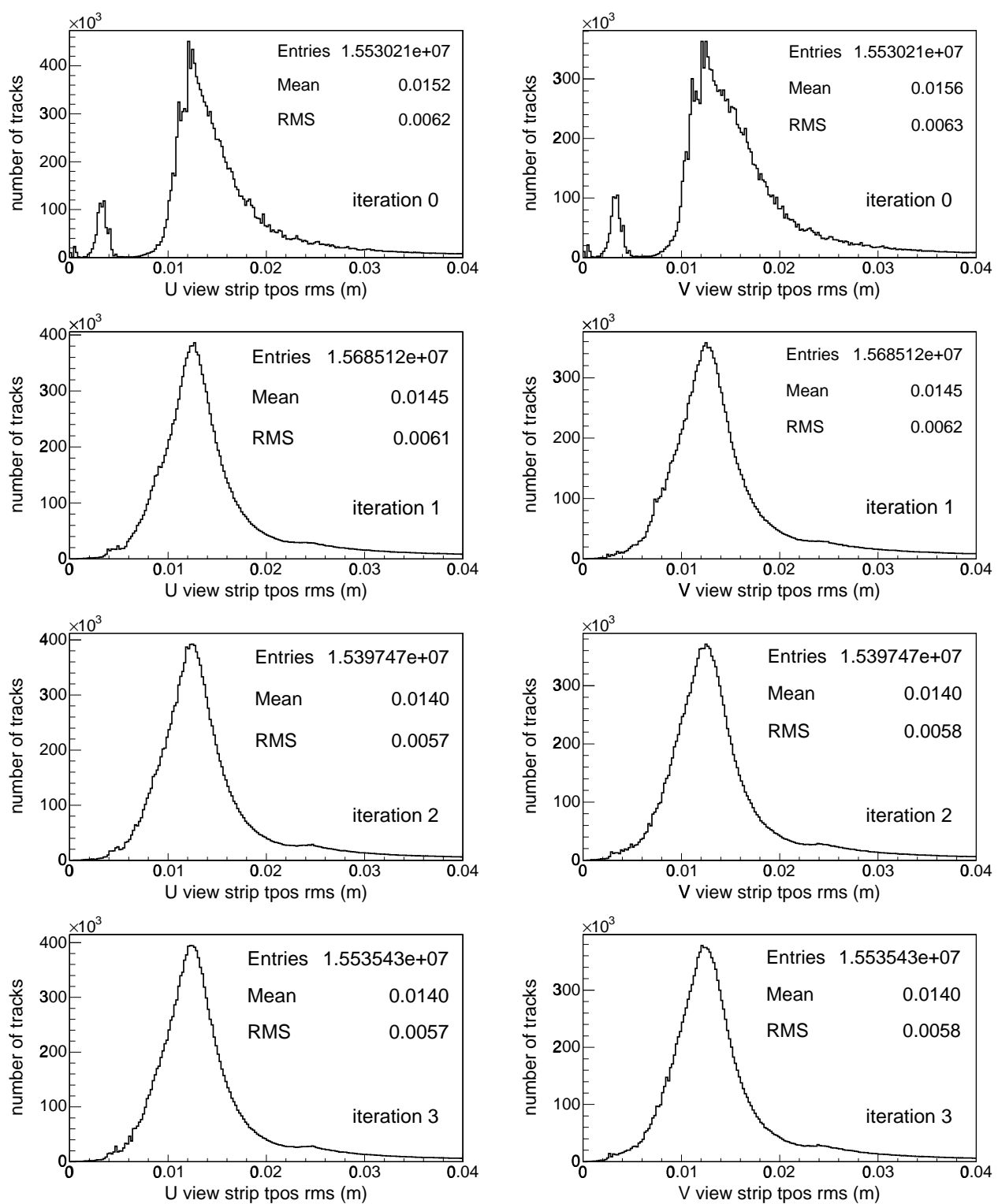

Figure 37: RMS of strip transverse positions around a best fit line to $2 \mathrm{~d}$ track. 